\title{
Atmospheric scattering of energetic electrons from near-Earth space
}

\author{
Vassilis Angelopoulos ( $\nabla$ vassilis@ucla.edu ) \\ University of California Los Angeles https://orcid.org/0000-0001-7024-1561 \\ Ethan Tsai \\ University of California Los Angeles
}

Colin Wilkins

University of California Los Angeles

\section{Xiaojia Zhang}

University of California Los Angeles

\section{Anton Artemyev}

University of California

Jiang Liu

University of California Los Angeles

\section{Andrei Runov}

University of California Los Angeles

\section{Laura Iglesias}

University of California Los Angeles

\section{Drew Turner}

The Aerospace Corp.

\section{Robert Strangeway}

Institute of Geophysics and Planetary Physics, UCLA

\section{Richard Wirz}

University of California Los Angeles

\section{Wen Li}

Boston University

\section{Lydia Adair}

University of California Los Angeles

\section{Ryan Caron}

University of California Los Angeles

\section{Maxwell Chung}

Johns Hopkins University, Applied Physics Laboratory

\section{Patrick Cruce}

Apple

Eric Grimes 
University of California Los Angeles

\section{Kathryn Hector}

Raytheon Space and Airborne Systems

\section{Michael Lawson}

University of California Los Angeles

\section{David Leneman}

University of California Los Angeles

\section{Emmanuel Masongsong}

University of California Los Angeles https://orcid.org/0000-0002-0266-6864

\section{Austin Norris}

University of California Los Angeles

\section{Cynthia Russell}

University of California Los Angeles

\section{Christopher Shaffer}

Tyvak Nano-Satellite Systems

\section{Jiashu Wu}

University of California Los Angeles

\section{Sharvani Jha}

University of California Los Angeles

James King

University of California Los Angeles

\section{Suyash Kumar}

University of California Los Angeles

\section{Kelly Nguyen}

University of California Los Angeles

\section{Michelle Nguyen}

University of California Los Angeles

\section{Akhil Palla}

University of California Los Angeles

\section{Alexa Roosnovo}

University of California Los Angeles

\section{Erica Xie}

University of California Los Angeles

\section{Rebecca Yap}

University of California Los Angeles

\section{Chanel Young}

University of California Los Angeles

\section{J. B. Blake}

The Aerospace Corporation 


\section{Nick Adair}

Millenium Space Systems

\section{Matthew Allen}

Northrop-Grumman Aerospace Systems

\section{Michael Anderson}

Lucid Motors

\section{Michael Arreola-Zamora}

Northrop-Grumman Aerospace Systems

Jessica Artinger

University of California Los Angeles

Jeffrey Asher

Johns Hopkins University, Applied Physics Laboratory

Donna Branchevsky

The Aerospace Corporation

\section{Michael Capitelli}

Millenium Space Systems

\section{Rommel Castro}

Raytheon Space and Airborne Systems

\section{Gary Chao}

Boeing

Nathan Chung

Tesla

Micah Cliffe

SpaceX

\section{Kyle Colton}

Planet Labs

\section{Cian Costello}

NovaSignal

\section{Danny Depe}

University of California Los Angeles

\section{Benjamin Domae}

University of California Los Angeles

\section{Sarah Eldin}

University of California Los Angeles

\section{Lauren Fitgibbon}

Tyvak Nano-Satellite Systems

\section{Alex Flemming}

Northrop-Grumman Aerospace Systems

Ian Fox 
University of California Los Angeles

\section{Duncan Frederick}

Millenium Space Systems

\section{Alex Gilbert}

University of California Los Angeles

\section{Anthony Gildemeister}

Northrop-Grumman Aerospace Systems

\section{Alexander Gonzalez}

University of California Los Angeles

\section{Brayden Hesford}

Jet Propulsion Laboratory

\section{Renee Krieger}

Mercedes-Benz Research and Development North America

\section{Kevin Lian}

Northrop-Grumman Aerospace Systems

Jason Mao

Epic Systems Corporation

Emmons McKinney

California State Polytechnic University, Pomona

\section{Jordan Miller}

University of California Los Angeles

\section{Matt Nuesca}

University of California Los Angeles

\section{Elisa Park}

University of California Los Angeles

\section{Carter Pedersen}

University of California Los Angeles

\section{Ziyuan Qu}

University of California Los Angeles

\section{Reuben Rozario}

SpaceX

\section{Erik Rye}

University of California Los Angeles

\section{Ryan Seaton}

University of California Los Angeles

\section{Akshaya Subramanian}

Northrop-Grumman Aerospace Systems

\section{Stephen Sundin}

Tyvak Nano-Satellite Systems 


\section{Aysen Tan}

Experior Laboratories

\section{Wynne Turner}

University of California Los Angeles

\section{Austin Villegas}

University of California Los Angeles

\section{Matt Wasden}

University of California Los Angeles

\section{Graham Wing}

University of California Los Angeles

\section{Cass Wong}

University of California Los Angeles

Anais Zarifian

Jet Propulsion Laboratory

\section{Gary Zhang}

University of California Los Angeles

\section{Physical Sciences - Article}

Keywords: Repopulation of the Magnetosphere, Radiation Belt Flux Evolution, Ozone Depletion, Global Atmospheric Circulation, Relativistic Electrons

Posted Date: March 12th, 2021

DOI: https://doi.org/10.21203/rs.3.rs-319558/v1

License: (c) (1) This work is licensed under a Creative Commons Attribution 4.0 International License. Read Full License 


\section{Atmospheric scattering of energetic electrons from near-Earth space}

2 V. Angelopoulos ${ }^{1 *}$, E. Tsai ${ }^{1}$, C. Wilkins ${ }^{1}$, X.-J. Zhang ${ }^{1}$, A. V. Artemyev ${ }^{1}$, J. Liu ${ }^{1}$, A. Runov ${ }^{1}$, L. 3 Iglesias $^{1}$, D. L. Turner ${ }^{2}$, R. J. Strangeway ${ }^{1}$, R. E. Wirz ${ }^{3}$, W. Li $^{4}$, L. Adair ${ }^{1,5}$, R. P. Caron ${ }^{1}$, M. Chung ${ }^{2}$, 4 P. Cruce ${ }^{6}$, E. Grimes ${ }^{1}$, K. Hector ${ }^{7}$, M. J. Lawson ${ }^{1}$, D. Leneman ${ }^{1}$, E. V. Masongsong ${ }^{1}$, A. Norris ${ }^{1}$, C.

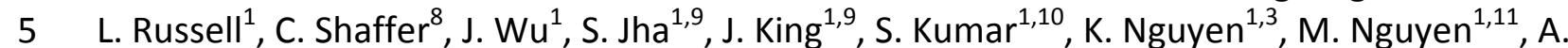
6 Palla $^{1,9}$, A. Roosnovo ${ }^{1,10}$, E. Xie ${ }^{1,11}$, R. Yap ${ }^{1,12}$, C. Young ${ }^{1,9}$, J. B. Blake ${ }^{13}$, N. Adair ${ }^{5}$, M. Allen ${ }^{14}$, M. 7 Anderson $^{15}$, M. Arreola-Zamora ${ }^{1,14}$, J. Artinger ${ }^{1,10}$, J. Asher ${ }^{2}$, D. Branchevsky ${ }^{13}$, M. R. Capitelli ${ }^{5}$, R.

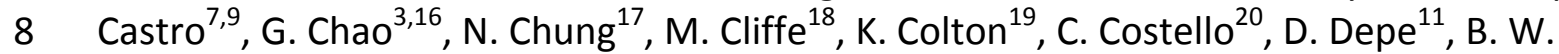
9 Domae $^{11}$, S. Eldin ${ }^{11}$, L. Fitzgibbon ${ }^{8}$, A. Flemming ${ }^{14}$, I. Fox ${ }^{3}$, D. M. Frederick ${ }^{5}$, A. Gilbert ${ }^{11}$, A. Gildemeister $^{14}$, A. Gonzalez ${ }^{9}$, B. Hesford ${ }^{21}$, R. Krieger ${ }^{22}$, K. Lian ${ }^{14}$, J. Mao ${ }^{23}$, E. McKinney ${ }^{24}$, J. P. Miller $^{9}$, M. Nuesca ${ }^{9}$, E. S. Y. Park ${ }^{25}$, C. E. Pedersen ${ }^{3}$, Z. Qu ${ }^{3}$, R. Rozario ${ }^{18}$, E. Rye ${ }^{11}$, R. Seaton ${ }^{1}, A$.

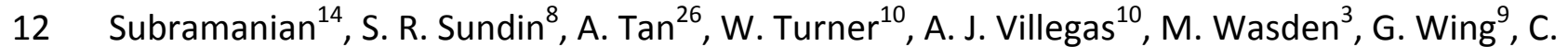
Wong $^{10}$, A. Zarifian ${ }^{21}$, G. Y. Zhang ${ }^{27}$

\section{4}

15

16

\section{Affiliations:}

1 Earth, Planetary, and Space Sciences Department, and Institute of Geophysics and Planetary Physics, University of California, Los Angeles, CA 90095

2 Johns Hopkins University Applied Physics Laboratory, Laurel, Maryland 20723

3 Mechanical and Aerospace Engineering Department, Henry Samueli School of Engineering, University of California, Los Angeles, CA 90095

4 Department of Astronomy and Center for Space Physics, Boston University, Boston, MA 02215

5 Millenium Space Systems, El Segundo, CA 90245

6 Apple Inc., Cupertino, CA 95014

7 Raytheon Space and Airborne Systems, El Segundo, CA 90245

8 Tyvak Nano-Satellite Systems, Inc., Irvine, CA 92618

9 Computer Science Department, Henry Samueli School of Engineering, University of California, Los Angeles, CA 90095

10 Physics and Astronomy Department, University of California, Los Angeles, CA 90095

11 Electrical and Computer Engineering Department, Henry Samueli School of Engineering, University of California, Los Angeles, CA 90095

12 Mathematics Department, University of California, Los Angeles, CA 90095

13 The Aerospace Corporation, El Segundo, CA 90245

14 Northrop Grumman Aerospace Systems, Redondo Beach, CA 90278

15 Lucid Motors, Newark, CA 94560

16 Boeing, El Segundo, CA 90245

17 Tesla, Palo Alto, CA 94306

18 SpaceX, Hawthorne, CA 90250

19 Planet Labs, San Francisco, CA 94107

20 NovaSignal, Los Angeles, CA 90064

21 Jet Propulsion Laboratory, Pasadena, CA 91109

22 Mercedes-Benz Research and Development North America, Long Beach, CA 90810

23 Epic Systems Corporation, Verona, WI 53593

24 California State Polytechnic University, Pomona, CA 91768

25 Economics Department, University of California, Los Angeles, CA 90095

26 Experior Laboratories, Oxnard, CA 93033

27 Qualcomm, San Diego, CA 92121

*Correspondence to: vassilis@ucla.edu 


\section{Summary paragraph:}

50 In near-Earth space, the magnetosphere, energetic electrons (tens to thousands of kiloelectron

51 volts) orbit around Earth, forming the radiation belts. When scattered by magnetospheric

52 processes, these electrons precipitate to the upper atmosphere, where they deplete ozone, a

53 radiatively active gas, modifying global atmospheric circulation. Relativistic electrons (those

54 above a few hundred kiloelectron volts), can reach the lowest altitudes and have the strongest

55 effects on the upper atmosphere; their loss from the magnetosphere is also important for

56 space weather. Previous models have only considered magnetospheric scattering and

57 precipitation of energetic electrons; atmospheric scattering of such electrons has not been

58 adequately considered, principally due to lack of observations. Here we report the first

59 observations of this process. We find that atmospherically-scattered energetic (relativistic)

60 electrons form a low-intensity, persistent "drizzle", whose integrated energy flux is comparable

61 to (greater than) that of the more intense but ephemeral precipitation by magnetospheric

62 scattering. Thus, atmospheric scattering of energetic electrons is important for global

63 atmospheric circulation, radiation belt flux evolution, and the repopulation of the

64 magnetosphere with lower-energy, secondary electrons.

Main article: Polar ozone exerts strong radiative forcing on global atmospheric

66 circulation by modifying temperature, winds, and waves in the upper atmosphere. Energetic

67 electrons from near-Earth space can reach the high-latitude mesosphere $(50-100 \mathrm{~km})$, where

68 they produce reactive odd nitrogen and hydrogen $\left(\mathrm{NO}_{\mathrm{x}}\right.$ and $\left.\mathrm{HO}_{\mathrm{x}}\right)$, ozone-destroying catalysts.

69 Nitrogen oxides can also descend to the stratosphere $(25-50 \mathrm{~km})$, where they become the most

70 important contributors to catalytic ozone destruction. Thus, energetic electron precipitation 
71 can affect the global ozone $c y c l e^{1,2,3}$ and global circulation significantly. Despite their

72 importance for modeling atmospheric circulation, energetic particles have not been adequately

73 incorporated into global atmospheric models ${ }^{4,5,6}$, resulting in large discrepancies between

74 model predictions and observations of vertical ozone profiles ${ }^{7}$. Magnetospheric energetic

75 electrons (especially relativistic ones) are also important for space weather, as they can damage

76 satellites and harm astronauts, particularly during magnetic storms ${ }^{8,9}$. Their fluxes, a delicate

77 balance of large contributions from transport, acceleration, and loss, vary so as to defy

78 predictability by modeling. These electrons can be trapped for hours to weeks in the outer

79 radiation belt, which is near the magnetic equator at geocentric distances of L=3-7 Earth radii.

80 Plasma waves $^{10}$ or extreme equatorial field-line curvature ${ }^{11}$ can scatter them, reducing their

81 velocity angle (pitch angle, $\alpha$ ) relative to the magnetic field, $\mathbf{B}$, to less than the loss cone angle

$82\left(\alpha<\alpha_{\mathrm{LC}}\right)$. This allows them to reach the mesosphere or stratosphere, where they collide, deposit

83 their energy and are lost from the magnetosphere. Although magnetospheric scattering has

84 been incorporated into radiation belt diffusion models ${ }^{12,13}$, because of lack of observations,

85 atmospheric scattering has not, resulting in significant model deficiencies ${ }^{14,15}$. Using the first

86 low-altitude $(\sim 410 \mathrm{~km})$, high-resolution (in both pitch angle and energy) observations of

87 energetic (50-5800keV) electrons by the ELFIN mission ${ }^{16}$, we report on atmospheric scattering

88 and its dependence on activity and location. We interpret upgoing electrons $\left(180^{\circ}-\alpha_{L C}<\alpha\right)$ at

89 some energy, E, as secondary electrons produced by atmospheric scattering of either trapped

$90\left(\alpha^{\sim 90^{\circ}}\right)$ or precipitating $\left(\alpha<\alpha_{\mathrm{LC}}\right)$ primary electrons of a greater energy. (Note: unless otherwise

91 stated, pitch angles are referenced to the northern hemisphere; for the southern hemisphere,

92 use their supplementary). We find that the net energetic (relativistic) electron energy flux 
93 precipitation from atmospheric scattering is comparable to (greater than) that from 94 magnetospheric scattering.

ELFIN, a dual CubeSat, polar-orbiting mission launched in 2018, has collected data from

$96>1000$ science zones (magnetically mapping to $L=2-15$ ), covering all local times and a wide

97 range of geomagnetic conditions. We use energetic particle detector instrument (EPDE) data

98 obtained by ELFIN-A (EL-A) from magnetic local times within \pm 4 hours of the noon-midnight

99 plane between 2019/09/01 2020/11/13 ( 700 science zones). The instrument has a single

100 square-aperture field-of-view $\left(\mathrm{FOV}=22^{\circ}\right)$. Spinning on a plane containing $\mathrm{B}$, once per spin

101 ( 3sec) it provides 15 energy channels (50keV-5800keV) of width $\Delta E / E \sim 40 \%$ and 16 spin-phase

102 sectors of width $\mathrm{SCW}=22.5^{\circ}$. Spin-phases are transformed to pitch-angles using the

103 international geophysical reference field (IGRF) model. At ELFIN's altitude, the loss cone is

$104 \alpha_{L C} \sim 67^{\circ} \pm 2^{\circ}$. Precipitating (downgoing) or atmospherically-scattered (upgoing) electrons were

105 measured when the detector's full width (FOV+SCW) was entirely within the loss cone or the

106 anti-loss cone, respectively. Trapped electrons had sector centers $\alpha=90^{\circ} \pm 11.25^{\circ}$.

107 Atmospheric scattering of precipitating electrons ("backscatter") should produce low 108 upgoing-to-downgoing ratios ${ }^{15}$. Thus, when magnetospherically scattered precipitation is

109 significant (relative to trapped fluxes), atmospheric backscatter should yield low upgoing-to-

110 downgoing flux ratios. Atmospheric scattering of (barely) trapped electrons should result in low

111 (relative to trapped) but balanced upgoing and downgoing fluxes at upper atmospheric

112 altitudes (upgoing-to-downgoing ratios $100 \%$ ). Thus, when magnetospherically-scattered

113 precipitation (and, consequently, its atmospheric backscattering) is low, atmospheric scattering 
114 of trapped fluxes can dominate, resulting in upgoing-to-downgoing flux ratios $100 \%$ at the

115 upper atmosphere. Under such conditions, the absence of magnetospheric scattering sites near

116 the equator should allow atmospherically-scattered upgoing energetic electrons from the

117 opposite hemisphere to be detected at the local hemisphere as downgoing, resulting in

118 upgoing-to-downgoing ratios $\sim 100 \%$ also at the satellite.

ELFIN-A observations of a northern, nightside (MLT 1) science zone (Figure 1a) during

120 an active time (the $D_{S T}$ index ${ }^{17}$ had a minimum of $-49 n T$ fourteen hours earlier ${ }^{18}$ ) confirms the

121 above expectations from atmospheric scattering. Significant downgoing fluxes $\left(\alpha<\alpha_{\mathrm{LC}}\right)$ are

122 evident between $\mathrm{L}=3.5$ and 6.5 (Figure $1 \mathrm{~b}-\mathrm{c}$ ). When the downgoing energy flux (precipitation)

123 was a large fraction of the trapped flux (as between 13:13:00 and 13:15:00UT, Figure 1f,h), the

124 upgoing $\left(180^{\circ}-\alpha_{L C}<\alpha\right)$ flux intensified, too (Figure $\left.1 d\right)$, but remained lower than the

125 precipitation (upgoing-to-downgoing ratio was a few percent, Figure 1i). Conversely, when the

126 precipitation was low, only a few percent of the trapped flux (as between 13:11:50 and

127 13:12:20UT, Figure 1f,h), the upgoing flux was also low (Figure 1d), but comparable to the

128 precipitation (up-to-down flux ratio 100\%, Figure 1i).

129 When intense precipitation from magnetospheric scattering occurs up to some

130 maximum energy, $E_{p m a x}$, atmospheric scattering above $E_{p m a x}$ is expected continue to be

131 dominated by atmospheric electron scattering at both hemispheres (upgoing-to-downgoing

132 ratio $100 \%)$, impervious to magnetospheric scattering and its atmospheric feedback below

$133 E_{p m a x}$. Indeed, this can be seen at $13: 12: 35 U T$, when the downgoing-to-perpendicular ratio

134 (Figure $1 \mathrm{~h})$ was elevated ( $50 \%)$ at $\mathrm{E}<\mathrm{E}_{\mathrm{pmax}} \sim 500 \mathrm{keV}$ : while the upgoing-to-downgoing ratio

135 (Figure 1i) was suppressed ( 30\%) at $E<E_{p m a x}$, it remained $\sim 100 \%$ at $E>E_{p m a x}$. Subsequently, as 
$136 E_{p m a x}$ increased progressively from $150 \mathrm{keV}$ to $800 \mathrm{keV}(13: 12: 45-13: 13: 15 \mathrm{UT}$, Figure $1 \mathrm{~h})$, the

137 energy where the upgoing-to-downgoing ratio transitioned from low $(<10 \%)$ to high $(>60 \%)$

138 values followed $E_{p m a x}$ (Figure 1i), as expected. Additional examples are shown in Extended Data

139 Figures 1 and 2 (nightside and dayside, respectively). Therefore, atmospheric scattering of

140 trapped fluxes is quantifiable and long-lasting, based on case studies.

Atmospheric scattering of intense, high-energy precipitation is also expected to create

142 copious backscattered electrons at $E<<E_{\text {pmax. }}$ Indeed, at 13:13:10-13:14:00UT, when the

143 downgoing-to-perpendicular ratio is high, 100\%, with $\mathrm{E}_{\mathrm{pmax}} \sim 1 \mathrm{MeV}$ (Figure $1 \mathrm{~h}$ ), the upgoing-to-

144 downgoing flux ratios are low ( 1-2\%) near $E_{\text {pmax }}$ (Figure 1i), but are significant (20-50\%) at 145 energies several times lower than $E_{p m a x}(50-150 k e V)$. Another example is in Extended Data 146 Figure 1 (13:47:15-13:47:30UT). Thus, atmospheric scattering of precipitation can also be a 147 significant source of energetic electrons in the magnetosphere, as previously suggested ${ }^{14,15}$.

149 as "energetic electron drizzle" and to atmospheric scattering of magnetospheric precipitation 150 (upward only) as "energetic electron backscatter". Upgoing secondary electrons can be 151 produced by either (generally both). Likewise, downgoing (or "precipitating") energetic 152 electrons can be from either downward drizzle (even from the opposite hemisphere) or 153 magnetospheric precipitation (originating from magnetospheric scattering even after 154 subsequent backscatter at the opposite hemisphere).

To further quantify the importance of atmospheric scattering, we employ broad-energy 156 flux channels LoE (50-430keV) and HiE (430-5800keV) in the upgoing, downgoing and 
157 perpendicular directions $\left(f_{u}, f_{d}, f_{\perp}\right.$, respectively), and statistically significant flux ratios within 158 each channel RLoE, RHiE $\left(f_{u} / f_{d}, f_{d} / f_{\perp}, f_{u} / f_{\perp}\right.$, with relative error $\left.<50 \%\right)$, as in Figure $1 j-m$ (and 159 Extended Data Figures 1I-L, 2I-L). These form the basis of our statistical analysis, below. 160 (Materials and Methods and Extended Data Figure 3 detail how these were constructed). Medians of the above ratios at the nightside (Figure $2 b, d$ ) exhibit L-shell variations

162 familiar from the nightside time series examined previously (Figure 1; Extended Data Figure 1):

163 at low L-shells, atmospheric drizzle dominates $\left(f_{d} / f_{\perp}<10 \% ; f_{u} / f_{d} \sim 70-100 \%\right) ;$ at high L-shells, 164 magnetospheric precipitation dominates $\left(f_{d} / f_{\perp}>40 \% ; f_{u} / f_{d} \sim 10 \%\right)$. The transition L-shell 165 decreases with geomagnetic activity (based on the Auroral Electrojet index $A E^{17,18}$ ). This is 166 consistent with an equatorward motion of the equatorward edge of the auroral oval (where 167 intense plasma waves and field-line scattering sites responsible for magnetospheric scattering 168 map), typical during active times ${ }^{19}$. At the dayside (Figure 2a,c), precipitation is dominated by 169 drizzle (as in Extended Data Figure 2). The statistical behavior of our dataset is thus expected to 170 be bimodal, with a drizzle-dominated subset at low L-shells and a magnetospheric precipitation171 dominated subset at high L-shells.

173 two peaks: The low-precipitation PDF peak $\left(\left(f_{d}+f_{u}\right) / f_{\perp} \sim 2-10 \%\right)$ has $f_{u} / f_{d} \sim 100 \%$, corresponds to 174 the low L-shells in Figures 1 and 2 (also Extended Data Figures 1 and 2), and is identified as 175 atmospheric drizzle. The high-precipitation PDF peak $\left(\left(f_{d}+f_{u}\right) / f_{\perp} \sim 100 \%\right)$ has $f_{u} / f_{d} \sim 7 \pm 3 \%$, 176 corresponds to the high L-shells in the above figures, and is identified as enhanced precipitation 177 mostly due to magnetospheric scattering. 
Precipitation of $50-430 \mathrm{keV}$ (LoE) electrons (Figure 3c) is dominated by intense

179 magnetospheric scattering (mostly by plasma waves), which overcomes the more common but 180 lower-intensity drizzle. Precipitation of 430-5800keV (HiE) and 50-5800keV (integral channel)

181 electrons is also dominated by magnetospheric scattering, but exhibits a significant

182 contribution from drizzle. Upgoing fluxes also exhibit a similar bimodal behavior (Figure 3b). At

183 all channels (LoE, HiE and integral), the drizzle peak $\left(\left(f_{d}+f_{u}\right) / f_{\perp} \sim 2-10 \%\right)$ dominates the upgoing

184 flux. However, the magnetospheric precipitation peak $\left(\left(f_{d}+f_{u}\right) / f_{\perp} \sim 100 \%\right)$, corresponding mostly

185 to backscatter (though likely some upward drizzle, too), also contributes significantly to the LoE

186 channel. These peaks and their properties remain similar when examined as a function of $f_{\perp}$

187 (Extended Data Figure 4) and geomagnetic activity and for the subset of the outer radiation belt $188(3<\mathrm{L}<7)$.

189 Evaluation of atmospheric scattering's net impact on precipitation starts from Table 1, 190 showing the measured upgoing-to-downgoing flux ratios, $r=\left\langle f_{u}\right\rangle \mid\left\langle f_{d}\right\rangle$, separately for the 191 nightside, dayside and combined. We see that $r \sim 45 \%$ for HiE and $r \sim 18 \%$ for the integral 192 channel.

Next, we recall that the downgoing flux contribution from scattering below the satellite

194 cannot be measured directly; it must be inferred. We note that the upgoing HiE and integral 195 flux (Figure $3 b$ ) are dominated by upward drizzle, $f_{a}$ (main peak, and likely a good part of the 196 secondary peak), which is up-down symmetric and occurs at both atmospheric feet of a field 197 line. It is therefore a good proxy for the downward drizzle arriving from the opposite 198 hemisphere. The measured downgoing flux for the HiE and integral channels (Figure 3c) is 
199 supplied by both magnetospheric precipitation, $f_{m}$, and downward drizzle from the opposite 200 hemisphere, $f_{a}$. Thus, at ELFIN, to zero order, we measure (Table 1): $r=\left\langle f_{u}\right\rangle \mid\left\langle f_{d}\right\rangle \sim f_{a} /\left(f_{a}+f_{m}\right)$. The 201 atmospheric scattering contribution to precipitation is $\sim 2 f_{a}$, the total precipitation at each 202 hemisphere is $\sim 2 \mathrm{f}_{\mathrm{a}}+\mathrm{f}_{\mathrm{m}}$, and the relative contribution of atmospheric scattering to precipitation 203 is $R=2 f_{a} /\left(2 f_{a}+f_{m}\right)$. If $x=2 f_{a} / f_{m}$ (atmospheric relative to magnetospheric scattering), using $r=x /(x+2)$ 204 and the measured values of $r$ (Table 1 ), we find $x=161 \%, R=1 /(1+x) \sim 62 \%$ for $\mathrm{HiE}$, and $x \sim 45 \%$, $205 \mathrm{R} \sim 31 \%$ for the integral channel. For the outer radiation belt $(3<\mathrm{L}<7)$ during all activity levels and 206 during only active times $\left(D_{S T}<-20 n T\right)$, we obtain (Extended Data Table 1$)$ similar values, though 207 somewhat reduced due to the increased relative contribution of magnetospheric precipitation 208 in those subsets.

209 Thus, atmospheric scattering contributes more than magnetospheric scattering to the 210 precipitation energy at relativistic energies (>430keV) and as much as $45 \%$ of the 211 magnetospheric precipitation at energies $>50 \mathrm{keV}$. Since relativistic electrons can reach the 212 upper/middle stratosphere, resulting in very efficient catalytic ozone depletions, and are also a 213 critical contributor to space weather, our results necessitate a factor of 2 upwards revision of 214 energy flux inputs in atmospheric models and energy flux losses in radiation belt models. 215 Moreover, during intense magnetospheric precipitation, the backscattered energetic electron 216 energy flux at low energies is a significant fraction of both precipitating and trapped flux. Thus,

217 atmospheric scattering can be also important for seeding the radiation belts with electrons and 218 for generating plasma waves; its effects need to be further quantified with observationally219 driven modeling. 
222 ELFIN data are available through http://elfin.igpp.ucla.edu.

223

Code availability statement

224

ELFIN mission data have been imported, analyzed, and plotted using corresponding

225 plug-ins to the open-source SPEDAS analysis platform ${ }^{20}$ (http://spedas.org). 
228 www.nature.com/nature.

Acknowledgments: We acknowledge support by NASA award NNX14AN68G (5/22/2014

230 - present) and NSF awards \# AGS-1242918 (9/24/2014-7/31/2019) and AGS-2019950 (July 2020

231 - present). We are grateful to NASA's CubeSat Launch Initiative program for successfully

232 launching the ELFIN satellites in the desired orbits under ELaNa XVIII. We thank the AFOSR for

233 early support of the ELFIN program under its University Nanosat Program, UNP-8 project,

234 contract \# FA9453-12-D-0285 (02/01/2013-01/31/2015). We also thank the California Space

235 Grant program for student support during the project's inception (2010-2014). We

236 acknowledge the hardware contributions and technical assistance of Mr. David Hinkley and The

237 Aerospace Corporation. We thank V. A. Sergeev, Y. A. Shprits and W. Greer for useful

238 discussions in the early stages of the program. We acknowledge the critical contributions of the

239 many volunteer ELFIN team members (more than 250 students). We thank Ms. J. Hohl for

240 editorial assistance with the manuscript. 


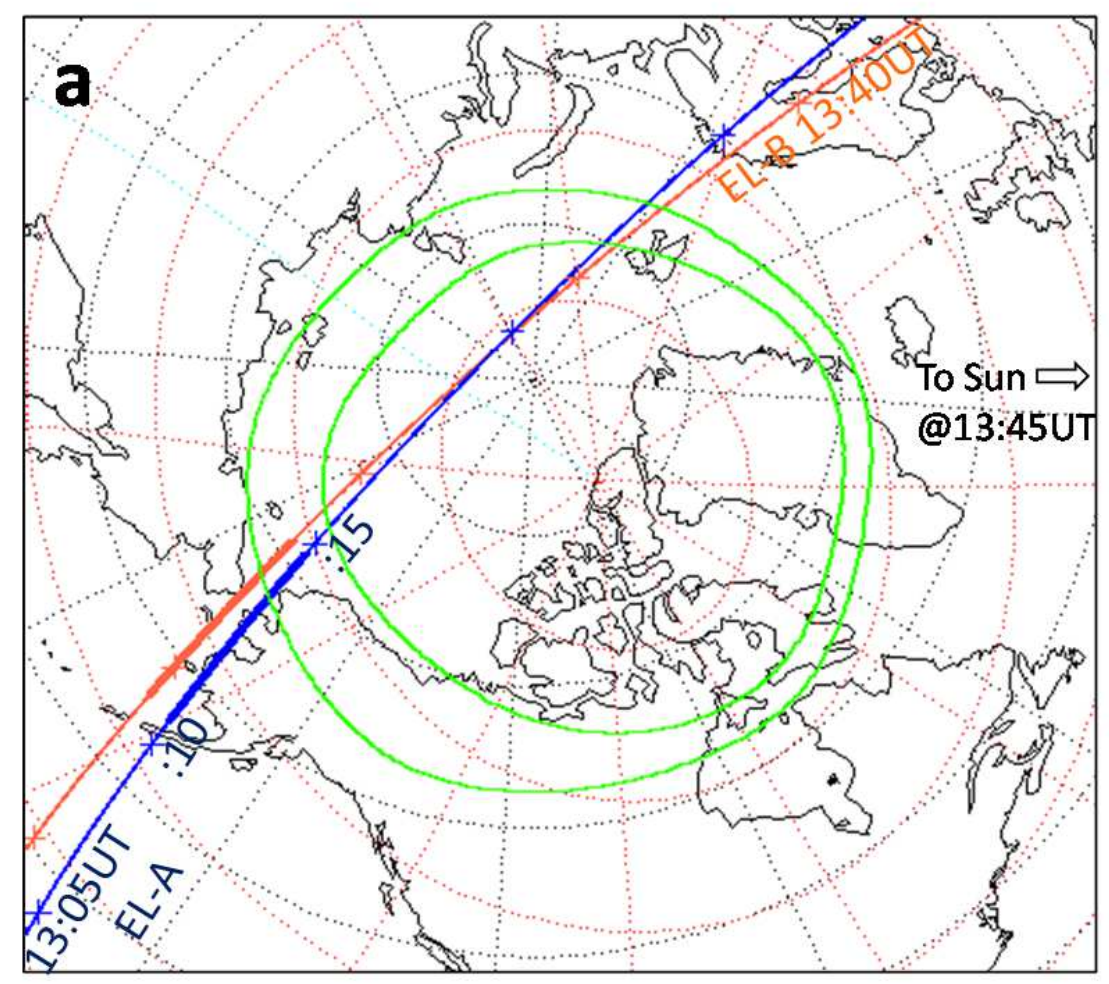

245

246 

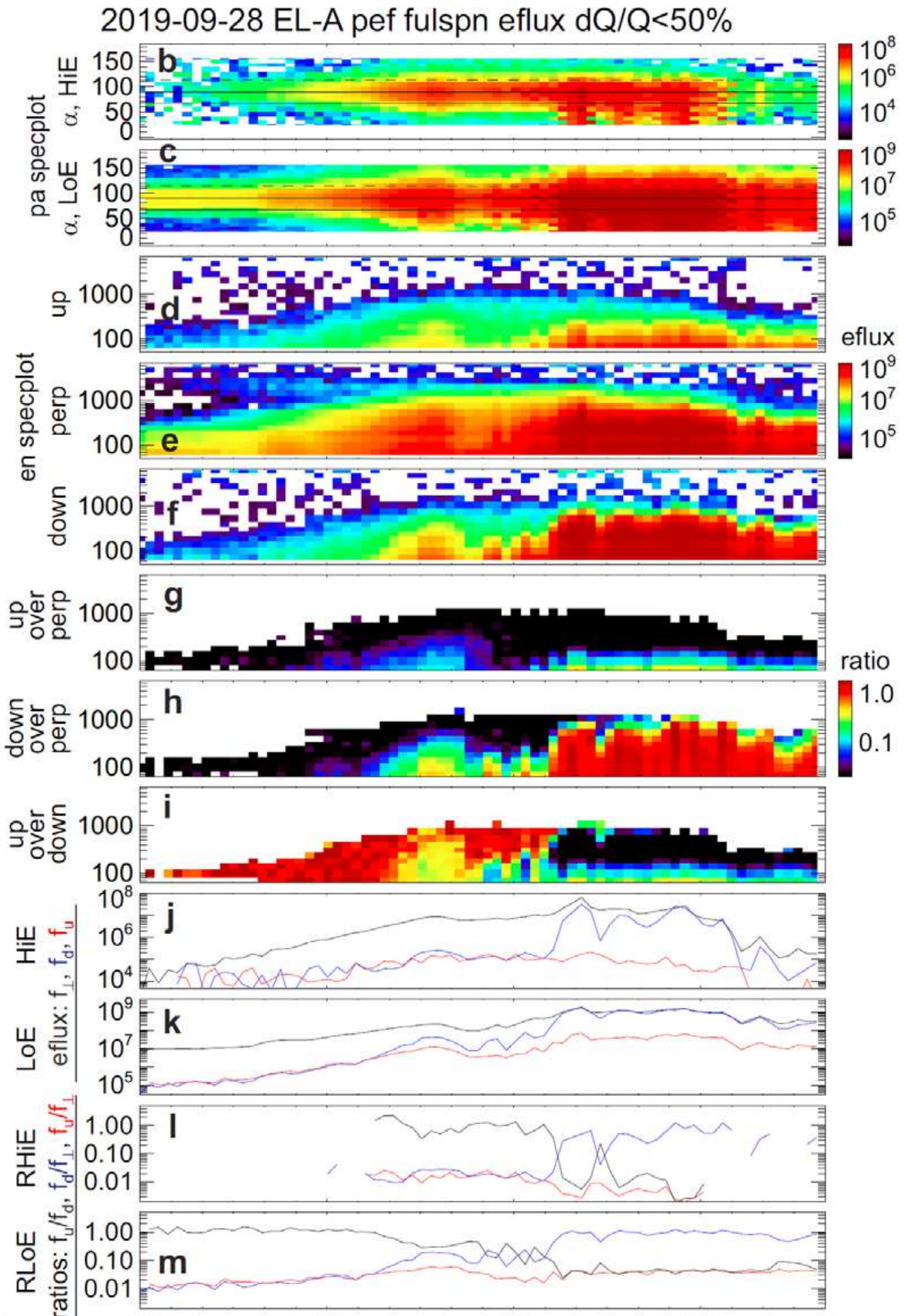

$\begin{array}{lrrrr}\text { L } & 3.3 & 4.0 & 4.9 & 6.2 \\ \text { MLT } & 1.5 & 1.3 & 1.1 & 0.8 \\ \text { MLA } & 55.2 & 58.7 & 62.1 & 65.4 \\ \text { hhmm } & 1311 & 1312 & 1313 & 1314\end{array}$


249 Figure 1 | ELFIN storm-time, nightside crossing of the outer radiation belt and auroral zone.

250 (a) EL-A satellite track geographic projections (fixed at 13:45UT) on 28 September 2019. Thick

251 lines represent times of data capture; 5-min intervals are indicated by crosses. Black (red)

252 dotted lines are geographic (corrected geomagnetic) meridians and parallels. Green lines are

253 nominal auroral oval boundaries. (b-c) Pitch-angle spectrograms of differential directional

254 energy flux ("energy flux" in keV/ $\mathrm{cm}^{2} \mathrm{~s}$ str MeV) in broad-energy electron channels HiE and LoE

255 (430-5800keV and 50-430keV energies, respectively). Bottom solid and upper dashed horizontal

256 lines in each spectrogram mark the loss cone $\left(\alpha=\alpha_{L C}\right)$ and anti-loss cone $\left(\alpha=180^{\circ}\right.$ - $\left.\alpha_{L C}\right)$; middle

257 solid line denotes $\alpha=90^{\circ}$. (d-f) Energy-time spectrograms of upgoing (within anti-loss

258 cone: $\alpha>180^{\circ}-\alpha_{L C}$ ), nearly-perpendicular to B (trapped), and downgoing (within loss

259 cone: $\alpha<\alpha_{\mathrm{LC}}$ ) electron energy flux. The energy ranges from $50 \mathrm{keV}$ to $5800 \mathrm{keV}$. (g-i) Energy-

260 time spectrograms of upgoing-to-perpendicular (up-to-perp, $f_{u} / f_{\perp}$ ), downgoing-to-perpendicular

261 (down-to-perp, $\left.f_{d} / f_{\perp}\right)$, and upgoing-to-downgoing $\left(f_{u} / f_{d}\right)$ electron energy flux. (j-k) Energy flux in

262 channels HiE and LoE (black: $f_{\perp}$; blue: $f_{d}$; red: $\left.f_{u}\right)$. (I-m) Ratios of energy flux in channels HiE and

263 LoE, respectively (black: $f_{u} / f_{d} ;$ blue: $f_{d} / f_{\perp} ;$ red: $f_{u} / f_{\perp}$ ). Annotations denote L-shell (L), dipole

264 magnetic local time (MLT), dipole magnetic latitude (MLA) and Universal Time (UT). 
Directional differential energy flux ratios

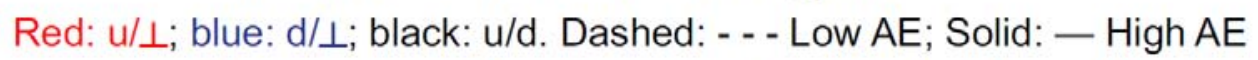

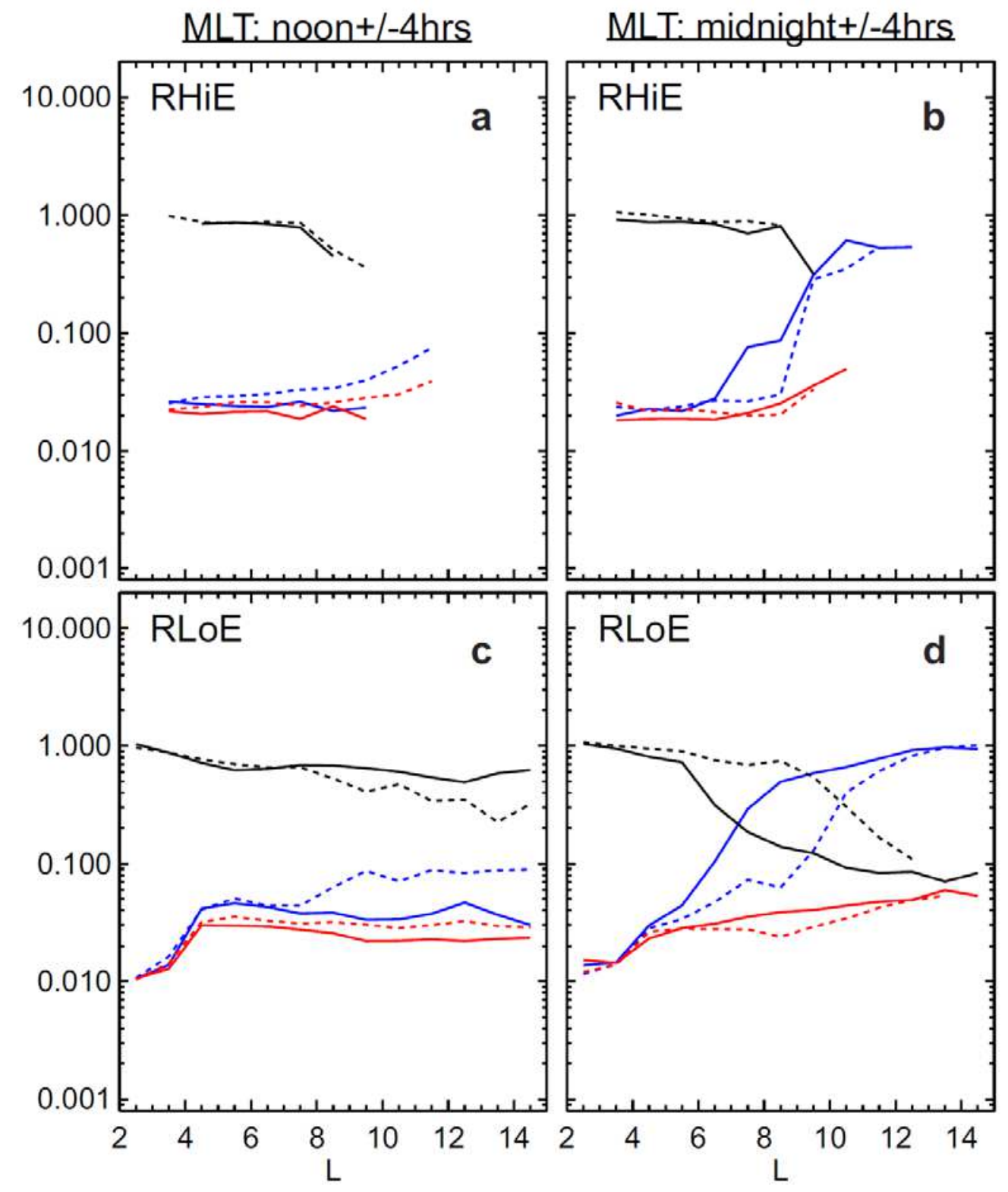


271 Figure 2 I Dependence of energy flux ratios on L-shell, local time, and activity.

272 (a-d) Medians of ratios: $f_{u} / f_{\perp}\left(\right.$ red), $f_{d} / f_{\perp}$ (blue), $f_{u} / f_{d}$ (black) as a function of L-shell. Top and 273 bottom panels: 1430 and 150 energy channels, respectively. Left and right panels: dayside and

274 nightside, respectively. Dashed and solid lines: data correspond to below and above the median

$275 A E$, respectively. ( $A E$ medians for dayside and nightside databases are: 110nT and 160nT, 276 respectively).

277

278 
Blue: LoE; Red: HiE; Black: 50-5800keV
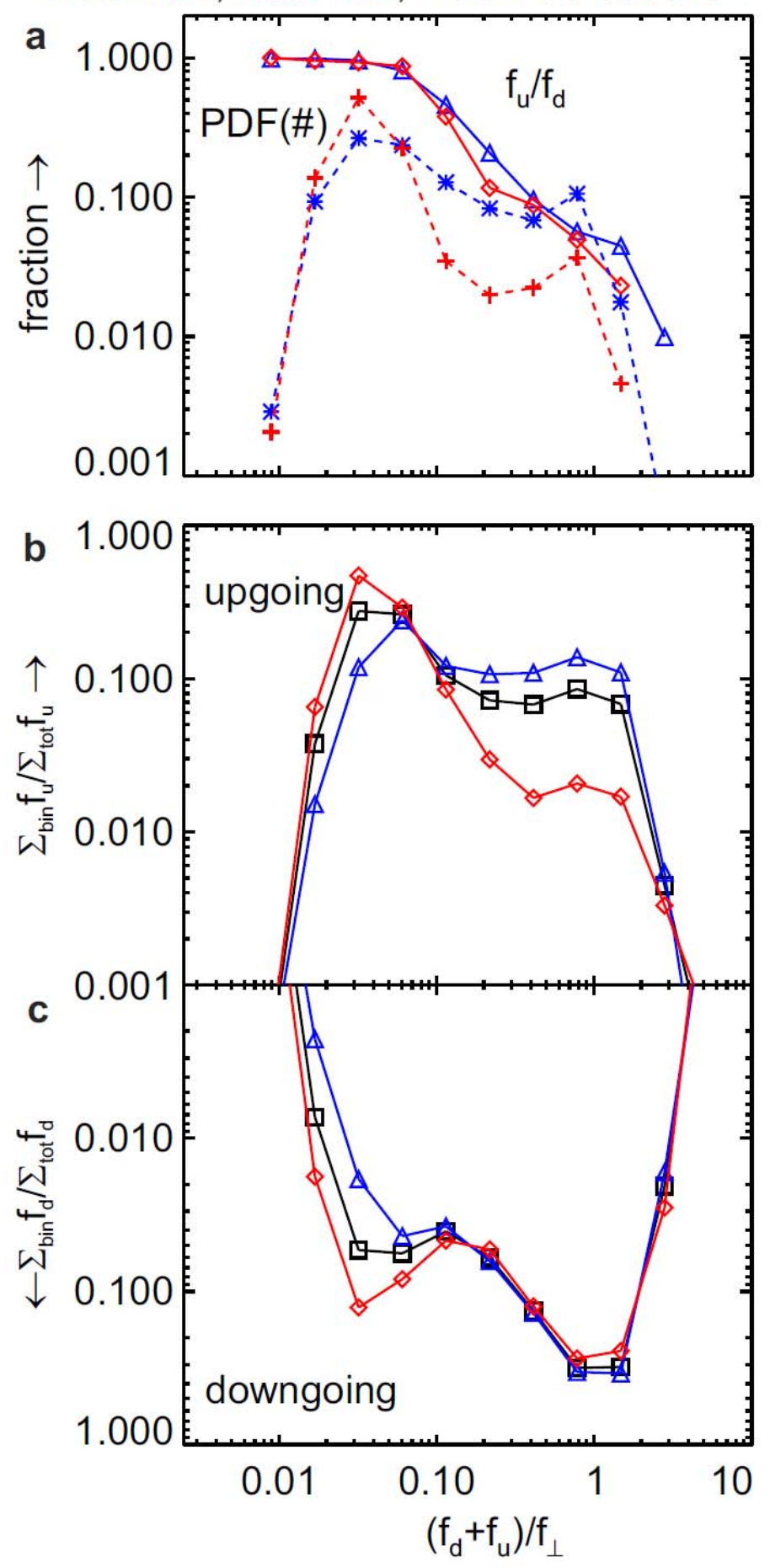
282 Figure 3 | Distribution of data, fluxes, and flux ratios as a function of loss-cone flux.

283 (a) Probability density functions of all data in the 150 and 1430 channels (blue stars and red 284 crosses, respectively) and medians of flux ratios $\left(f_{u} / f_{d}\right)$ for these channels (blue triangles and red 285 diamonds, respectively). (b) Relative contribution to net upward flux within the 150 and 1430 286 broad differential broad energy channels (blue triangles and red diamonds, respectively) and 287 within the summed energy channel representing the total energy flux measured by the 288 detector, i.e., at energies $50 \mathrm{keV}-5.8 \mathrm{MeV}$ (black squares). (c) Same as in (c) but for the 289 downward flux. Two-dimensional versions of several of these distributions, also plotted against 290 the perpendicular energy flux, are shown in Extended Data Figure 4. 


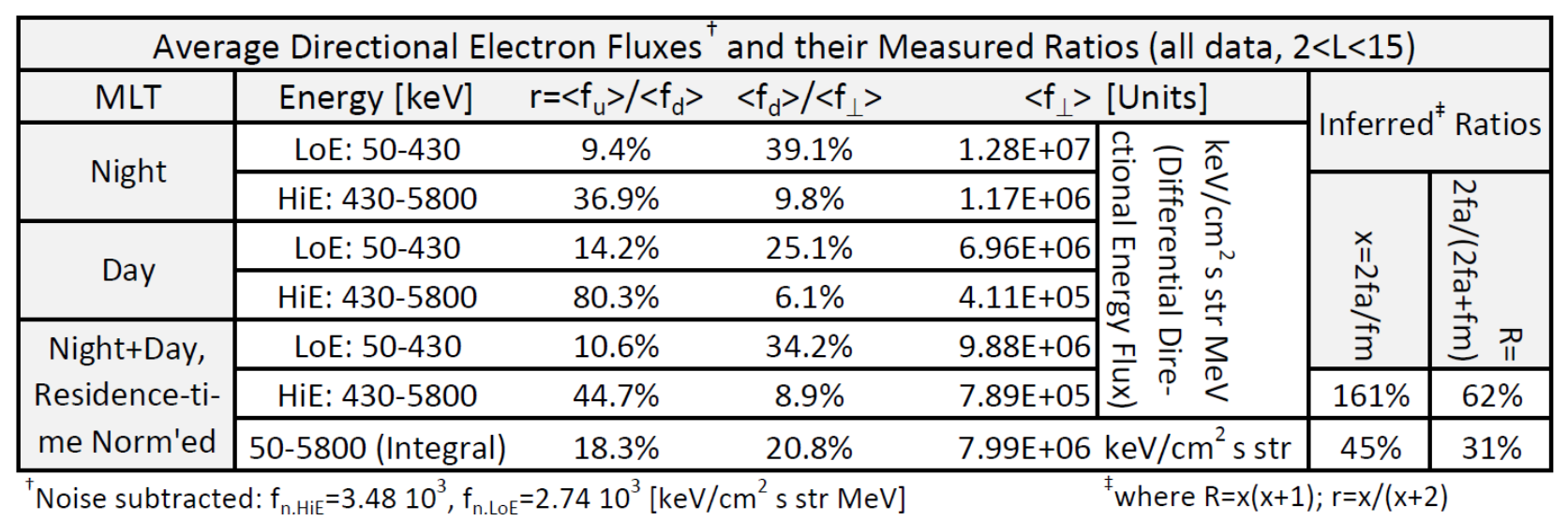

Table 1 | Differential and integral directional energy fluxes and ratios under all geomagnetic

297 than medians of ratios have been used, to accurately characterize total energy flux ratios.

298 Night+Day ratios were computed directly from the numbers above them, assuming equal 299 satellite residence time at day and night. Bottom row, which represents the integral directional 300 energy flux channel (50-5800keV), was computed directly from the rows above it. $f_{u}$ and $f_{d}$ are 301 upgoing and downgoing fluxes, $<>$ represents average, and $f_{a}$ and $f_{m}$ are the measured 302 contributions to precipitation from atmospheric scattering and magnetospheric scattering. The 303 ratios $x$ and $\mathrm{R}$ are the inferred net contributions to precipitation from atmospheric scattering 304 relative to magnetospheric scattering and relative to the total precipitation, respectively. 
Supplementary Information:

307 Methods

308

Extended Data Figures 1 - 4

309

Extended Data Table 1

310

311

312 
${ }^{1}$ Jackman, C. H., Frederick, J.E. \& Stolarski, R.S. Production of odd nitrogen in the stratosphere and mesosphere: An intercomparison of source strengths. J. Geophys. Res., Oceans. 85, C12, 7495-7505 https://doi.org/10.1029/JC085iC12p07495 (1980).

2 Thorne, R.M. The importance of energetic particle precipitation on the chemical composition of the middle atmosphere. PAGEOPH 118, 128-151 https://doi.org/10.1007/BF01586448 (1980).

${ }^{3}$ Randall, C.E. et al. Stratospheric effects of energetic particle precipitation in 2003-2004. Geophys. Res. Lett. 32, 5, https://doi.org/10.1029/2004GL022003 (2005).

${ }^{4}$ Baker, D.N. et al. Highly relativistic magnetospheric electrons: A role in coupling to the middle atmosphere? Geophys. Res. Lett. 14, 10 https://doi.org/10.1029/GL014i010p01027 (1987).

${ }^{5}$ Mirinova, I. A. et al. Ionization of the Polar Atmosphere by Energetic Electron Precipitation Retrieved From Balloon Measurements. Geophys. Res. Lett. 46, 2, 990-996 https://doi.org/10.1029/2018GL079421 (2019).

${ }^{6}$ Funke, B. et al. HEPPA-II model-measurement intercomparison project: EPP indirect effects during the dynamically perturbed NH winter 2008-2009. Atmos. Chem. Phys., 17, 5, 3573-3604 https://doi.org/10.5194/acp-17-3573-2017 (2017).

${ }^{7}$ Randall, C.E. et al. Simulation of energetic particle precipitation effects during the 2003-2004 Arctic winter. J. Geophys. Res. Space Phys. 120, 6, 5035-504 ,https://doi.org/10.1002/2015JA021196 (2016).

${ }^{8}$ Gonzales, W.D. et al. What is a geomagnetic storm? J. Geophys. Res. 99, A4, 5771-5792 doi:10.1029/93JA02867 (1994).

${ }^{9}$ Baker, D. N., et al., Space Weather Effects in the Earth's Radiation Belts, Space Sci. Rev., 214, 17, https://doi.org/10.1007/s11214-017-0452-7 (2018).

${ }^{10}$ Schulz, M. \& Lanzerotti, L.J. Particle Diffusion in the Radiation Belts in Physics and Chemistry in Space (ed. vol. 7, Roederer, J.G.)(Springer-Verlag, New York) https://doi.org/10.1007/978-3-642-65675-0 (1974).

${ }^{11}$ Imhof, W.L., Reagan, J.B. \& Gaines, E.E., Fine-scale spatial structure in the pitch angle distributions of energetic particles near the midnight trapping boundary. J. Geophys. Res. Space Phys. 82, 32, 5215-5221 https://doi.org/10.1029/JA082i032p05215 (1977).

12 Shprits, Y.Y., Subbotin, D. \& Binbin, N., Evolution of electron fluxes in the outer radiation belt computed with the VERB code. J. Geophys. Res., Space Phys. 114, A11 https://doi.org/10.1029/2008JA013784 (2009).

${ }^{13}$ Glauert, S. A., Horne, R.B., \& Meredith, N.P. Three-dimensional electron radiation belt simulations using the BAS Radiation Belt Model with new diffusion models for chorus, plasmaspheric hiss, and lightning-generated whistlers. J. Geophys. Res., Space Phys., 119, 1, 268-289 https://doi.org/10.1002/2013JA019281 (2014).

${ }^{14}$ Khazanov, G. V., Glocer, A. \& Chu, M. The Formation of Electron Heat Flux in the Region of Diffuse Aurora. J. Geophys. Res., Space Phys. 125, 8, https://doi.org/10.1029/2020JA028175 (2020). 
${ }^{15}$ Marshall, R. A., \& Bortnik, J. Pitch Angle Dependence of Energetic Electron Precipitation: Energy Deposition, Backscatter, and the Bounce Loss Cone. J. Geophys. Res., Space Phys. 123, 3, 2412-2423 https://agupubs.onlinelibrary.wiley.com/doi/full/10.1002/2017JA024873 (2018).

${ }^{16}$ Angelopoulos, V., et al. The ELFIN Mission, Space Sci. Rev. 216, 103 https://doi.org/10.1007/s11214-020-00721-7 (2020).

17 Mayaud, P. N. Derivation, meaning, and use of geomagnetic indices. Geophys. Monogr. Ser. 22, 154 pp., American Geophysical Union, doi:10.1029/GM022 (1980).

${ }^{18}$ http://wdc.kugi.kyoto-u.ac.jp/

19 Yokoyama, N., Kamide, Y., \& Miyaoka, H., The size of the auroral belt during magnetic storms. Ann. Geophys. 16, 566-573 https://doi.org/10.1007/s00585-998-0566-z (1998).

${ }^{20}$ Angelopoulos, V., et al., The Space Physics Environment Data Analysis System (SPEDAS). Space Sci. Rev. 215, 9 https://doi.org/10.1007/s11214-018-0576-4 (2019). 


\section{Atmospheric scattering of energetic electrons from near-Earth space}

V. Angelopoulos ${ }^{1 *}$, E. Tsai ${ }^{1}$, C. Wilkins ${ }^{1}$, X.-J. Zhang ${ }^{1}$, A. V. Artemyev ${ }^{1}$, J. Liu ${ }^{1}$, A. Runov ${ }^{1}$, L. Iglesias $^{1}$, D. L. Turner ${ }^{2}$, R. J. Strangeway ${ }^{1}$, R. E. Wirz ${ }^{3}$, W. Li ${ }^{4}$, L. Adair ${ }^{1,5}$, R. P. Caron ${ }^{1}$, M. Chung $^{2}$, P. Cruce ${ }^{6}$, E. Grimes ${ }^{1}$, K. Hector ${ }^{7}$, M. J. Lawson ${ }^{1}$, D. Leneman ${ }^{1}$, E. V. Masongsong ${ }^{1}$, A. Norris $^{1}$, C. L. Russell ${ }^{1}$, C. Shaffer ${ }^{8}$, J. Wu ${ }^{1}$, S. Jha, ${ }^{1,9}$, J. King ${ }^{1,9}$, S. Kumar ${ }^{1,10}$, K. Nguyen ${ }^{1,3}$, M. Nguyen $^{1,11}$, A. Palla ${ }^{1,9}$, A. Roosnovo ${ }^{1,10}$, E. Xie ${ }^{1,11}$, R. Yap ${ }^{1,12}$, C. Young ${ }^{1,9}$, J. B. Blake ${ }^{13}$, N. Adair $^{5}$, M. Allen ${ }^{14}$, M. Anderson ${ }^{15}$, M. Arreola-Zamora ${ }^{1,14}$, J. Artinger, ${ }^{10}$, J. Asher $^{2}$, D. Branchevsky $^{13}$, M. R. Capitelli ${ }^{5}$, R. Castro ${ }^{7,9}$, G. Chao $^{3,16}$, N. Chung ${ }^{17}$, M. Cliffe ${ }^{18}$, K. Colton ${ }^{19}$, C. Costello ${ }^{20}$, D. Depe ${ }^{11}$, B. W. Domae ${ }^{11}$, S. Eldin $^{11}$, L. Fitzgibbon ${ }^{8}$, A. Flemming ${ }^{14}$, I. Fox ${ }^{3}$, D. M. Frederick ${ }^{5}$, A. Gilbert ${ }^{11}$, A. Gildemeister ${ }^{14}$, A. Gonzalez ${ }^{9}$, B. Hesford ${ }^{21}$, R. Krieger ${ }^{22}$, K. $\mathrm{Lian}^{14}$, J. Mao ${ }^{23}$, E. McKinney ${ }^{24}$, J. P. Miller ${ }^{9}$, M. Nuesca ${ }^{9}$, E. S. Y. Park ${ }^{25}$, C. E. Pedersen ${ }^{3}$, Z. $\mathrm{Qu}^{3}$, R. Rozario ${ }^{18}$, E. Rye ${ }^{11}$, R. Seaton ${ }^{1}$, A. Subramanian ${ }^{14}$, S. R. Sundin ${ }^{8}$, A. Tan ${ }^{26}$, W. Turner ${ }^{10}$, A. J. Villegas ${ }^{10}$, M. Wasden ${ }^{3}$, G. Wing ${ }^{9}$, C. Wong ${ }^{10}$, A. Zarifian ${ }^{21}$, G. Y. Zhang ${ }^{27}$

\section{Affiliations:}

1 Earth, Planetary, and Space Sciences Department, and Institute of Geophysics and Planetary Physics, University of California, Los Angeles, CA 90095

2 Johns Hopkins University Applied Physics Laboratory, Laurel, Maryland 20723

3 Mechanical and Aerospace Engineering Department, Henry Samueli School of Engineering, University of California, Los Angeles, CA 90095

4 Department of Astronomy and Center for Space Physics, Boston University, Boston, MA 02215

5 Millenium Space Systems, El Segundo, CA 90245

6 Apple Inc., Cupertino, CA 95014

7 Raytheon Space and Airborne Systems, El Segundo, CA 90245

8 Tyvak Nano-Satellite Systems, Inc., Irvine, CA 92618

9 Computer Science Department, Henry Samueli School of Engineering, University of California, Los Angeles, CA 90095

10 Physics and Astronomy Department, University of California, Los Angeles, CA 90095

11 Electrical and Computer Engineering Department, Henry Samueli School of Engineering, University of California, Los Angeles, CA 90095

12 Mathematics Department, University of California, Los Angeles, CA 90095

13 The Aerospace Corporation, El Segundo, CA 90245

14 Northrop Grumman Aerospace Systems, Redondo Beach, CA 90278

15 Lucid Motors, Newark, CA 94560

16 Boeing, El Segundo, CA 90245

17 Tesla, Palo Alto, CA 94306

18 SpaceX, Hawthorne, CA 90250

19 Planet Labs, San Francisco, CA 94107

20 NovaSignal, Los Angeles, CA 90064

21 Jet Propulsion Laboratory, Pasadena, CA 91109

22 Mercedes-Benz Research and Development North America, Long Beach, CA 90810

23 Epic Systems Corporation, Verona, WI 53593

24 California State Polytechnic University, Pomona, CA 91768

25 Economics Department, University of California, Los Angeles, CA 90095

26 Experior Laboratories, Oxnard, CA 93033

27 Qualcomm, San Diego, CA 92121

49 *Correspondence to: vassilis@ucla.edu 
50 This file includes:

51

52

Methods

(pages 3-6)

53

Extended Data Figures $1-4$

(pages 7-15)

54

Extended Data Table 1

(page 16)

55

56

57

58

59

60 


\section{M1. Statistical significance of loss cone fluxes and their ratios}

Although the EPDE's side-penetrating radiation is insignificant thanks to high shielding

65 and coincidence $\operatorname{logic}{ }^{16}$, counting statistics must still be utilized to guarantee a robust signal-to-

66 noise ratio. Poisson statistics govern detector counts; the relative error dQ/Q of any quantity $Q$

67 proportional to the count rate (such as the energy flux) is $1 / \sqrt{ } N$, where $N$ is the total number of

68 counts in the measurement. To determine it, we obtain the net raw number of counts, N, that

69 contributed to each measurement (e.g., Q may be the average energy flux in two or three

70 sectors within the loss cone) and carry this information in the data processing along with the

71 measurement. For derived products, such as integral or average energy flux, we then use error

72 propagation formulas to compute the error for each quantity at every time step. An error

73 tolerance of $\mathrm{dQ} / \mathrm{Q}<50 \%$ for a data point would thus require that at least $\mathrm{N}=4$ counts

74 contributed to that measurement of $Q$.

75 Electronic noise, which also exists in the measurements, can be recognized as random,

76 low-flux pixels at high energies in the energy spectra in Figures 1d-f (and also in Extended Data

77 Figures $1 \mathrm{C}-\mathrm{E}$ and $2 \mathrm{C}-\mathrm{E}$ ). Most often each pixel corresponds to one count. This electronic noise is

78 readily eliminated by the aforementioned criterion $d Q / Q<50 \%$ when applied to derived

79 products, such as flux ratio spectrograms (Figures 1g-i; Extended Data Figures 1F-H and 2F-H),

80 or to the time-series ratios of directional broad-energy channels $\mathrm{HiE}$ and LoE. The very low

81 contribution of electronic noise to the measurement can be readily assessed from data

82 collected at the magnetic equator, below the inner belt, when no geophysical signal is present.

83 From such data we determined that electronic noise contributes $f_{n, H i E}=3.48 \times 10^{3}$ 
$84 \mathrm{keV} / \mathrm{cm}^{2} \mathrm{~s} \cdot \mathrm{str} \cdot \mathrm{MeV}$ and $\mathrm{f}_{\mathrm{n}, \mathrm{LoE}}=2.74 \times 10^{3} \mathrm{keV} / \mathrm{cm}^{2} \mathrm{~s} \cdot \mathrm{str} \cdot \mathrm{MeV}$ to the energy flux in the two energy

85 channels, HiE and LoE, respectively. We subtracted this noise from measurements in our 86 statistics if they had not already been subjected to the counting statistics threshold (e.g., dQ/Q

$87<50 \%$ or similar) that automatically rejects electronic noise.

To demonstrate that noise does not affect our loss-cone measurements, we show in

89 Extended Data Figure 3A the energy flux spectra as a function of pitch angle, averaged over 11

90 spins during the moderate precipitation interval, 13:12:17-13:12:50 UT in Figure 1. A pitch-

91 angle $\alpha=0^{\circ}$ corresponds to downgoing electrons, and vertical long-dashed lines denote the loss

92 cone (short-dashed lines denote the anti-loss cone). The dashed colored lines, mirror-images of

93 the downgoing fluxes about the pitch angle, $\alpha=0^{\circ}$, enable direct comparison of upgoing (solid)

94 and downgoing (dashed) lines at the same energy (color) in the raw data. The upgoing-to-

95 downgoing flux ratio in the loss cone thus can be estimated from Extended Data Figure $3 \mathrm{~A}$ to be

96 about $30 \%$ at low energies (warm colors, higher fluxes) and to approach $100 \%$ at high energies

97 (cold colors, lower fluxes). The horizontal dashed line represents a flux corresponding to 10

98 counts, i.e., a relative error of $d Q / Q ~ \sim 30 \%$ (here $Q$ is the energy flux in each sector, centered at

99 one distinct pitch angle). Below that horizontal dashed line, the data points fluctuate

100 considerably, consistent with statistical noise, but above it, the data points vary smoothly in

101 pitch angle. Our conclusions regarding ratio evolution are drawn from fluxes that are well

102 above the horizontal dashed line, based on dQ/Q criteria, and therefore are statistically

103 significant.

$104 \quad$ Upgoing-to-perpendicular and upgoing-to-downgoing ratios of flux averages derived

105 from Extended Data Figure 3, Panel A, are plotted in Panel C; for convenience, these are plotted 
106 on the left and right halves of the panel, respectively. Only the ratios with dQ/Q<30\% are

107 plotted; the absolute error based on the number of counts for each ratio is demarcated above

108 and below each point by a vertical bar. This restriction on counting statistics also eliminates

109 electronic noise, as discussed earlier. The upgoing-to-downgoing ratio of the average fluxes

110 (right half of Panel B) exhibits the behavior already surmised from the raw data in Panel A:

111 within the loss cone, it is low at low energies (warm colors) but it approaches $100 \%$ at

112 increasing energies (cold colors). This behavior is also consistent with the plots of instantaneous

113 (one per spin) flux ratios in Figure 1i;, Figure 1l,m; and their equivalent panels in Extended

114 Figures 1 and 2. It shows that statistical or electronic noise has been duly eliminated and does

115 not interfere with our ability to obtain statistically significant fluxes and flux ratios.

\section{M2. Purity of loss-cone flux}

117 For each sector in spin phase, the detector's finite geometric field of view $\left(22^{\circ}\right)$ and

118 finite accumulation time in spin phase $\left(22.5^{\circ}\right)$ result in a full width of $44.5^{\circ}$ and full width at

119 half-max of the contribution to the sector's flux of $33.25^{\circ}$. We rotate the two-dimensional

120 angular detector view (originally in polar and azimuthal angles in spacecraft geometric

121 coordinates) into field-aligned (pitch-angle and gyro-phase) coordinates and collapse it into 1D

122 pitch-angle space at every spin. This results in a smaller full width in pitch-angle space (as low

123 as $22^{\circ}$ ). We ensure that the viewing windows of the sectors we rely upon to produce the net

124 loss-cone flux are all inside the loss cone, up to the vertices of those windows.

125 To demonstrate the result of this mapping process, we show the fields of view of all 126 sectors during all spins in Extended Data Figure 3, Panel B (11 spins x 16 sectors are 127 overplotted in that panel, but the spin-to-spin variation is imperceptible, as the magnetic field 
128 direction does not change appreciably in the time interval considered). The full width is the thin

129 horizontal line, and the full width half-max is the thick horizontal line. The detector measures

130 particles arriving from the exact edge of the thick line for only $50 \%$ of the full sector

131 accumulation time (as opposed to $100 \%$ at the center). The contribution to the sector's average

132 flux from pitch angles outside the thick line decreases linearly to $0 \%$ at the pitch angles at two

133 edges of the thin line.

134 For a bin's measurements to be counted in the upgoing or downgoing flux, we require

135 that its full width be in the nominal loss cone (or anti-loss cone). Any contribution of the loss

136 cone's finite edge to the total flux is therefore attenuated by the limited time the detector

137 spends in that direction $\left(<1 / 32\right.$ of the sector's flux contribution arises from a $5.6^{\circ}$ angle next to

138 its edge) and by the contribution of other sectors well inside the loss cone. In Extended Data

139 Figure 3, Panels B and C, four sectors contribute to the downgoing flux and four to the upgoing

140 flux, the four closest to $\alpha=0^{\circ}$ and $\alpha=180^{\circ}$, respectively. Their upgoing-to-perperpendicular and

141 upgoing-to-downgoing ratios exhibit a smooth variation with $\alpha$. Our conclusions on ratios

142 drawn from those four sectors are consistent with the behavior of the two sectors with edges

143 farthest from the loss cone $\left(>15^{\circ}\right)$. 
2020-09-29 EL-A pef fulspn eflux dQ/Q $<50 \%$
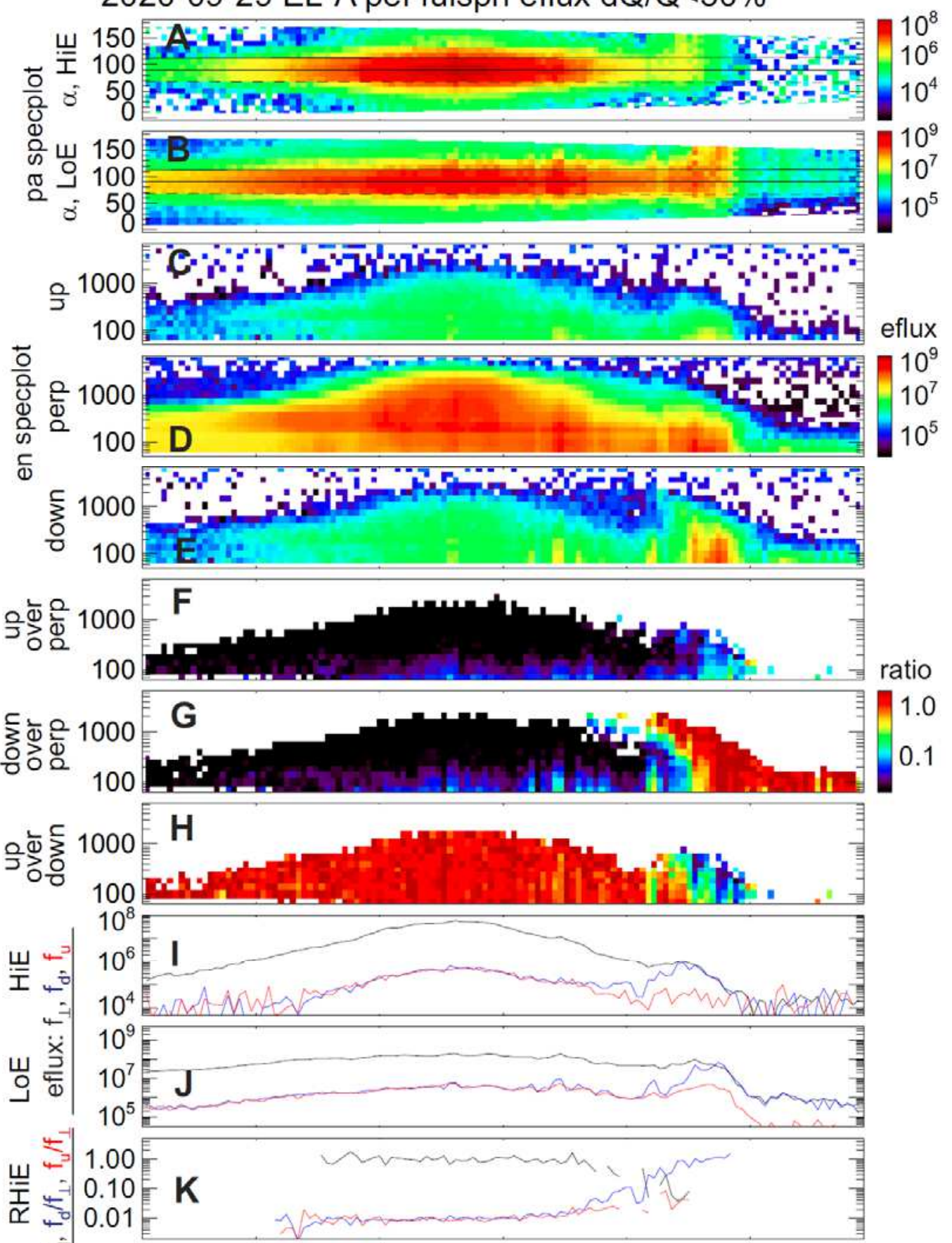

MLA

hhmm

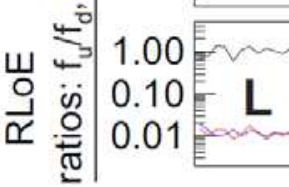

MLT

3.9

$-58.2$

0144
6.1

23.1

$-65.2$
1.0

0.1 
147 Extended Data Figure 1 | ELFIN-A nonstorm-time, nightside crossing of the outer radiation

148 belt and auroral zone. Format of Panels $A-L$ is identical to that of Panels b-m in Figure 1. (A-B)

149 Pitch-angle spectrograms of differential directional (broad) electron energy flux channels HiE 150 and LoE (430-5800keV and 50-430keV, respectively). Upper solid and bottom dashed horizontal

151 lines: loss cone $\left(\alpha=\alpha_{L C}\right)$ and anti-loss cone $\left(\alpha=180^{\circ}-\alpha_{L C}\right)$; middle solid line: $\alpha=90^{\circ}$. (C-E) Energy-

152 time spectrograms of upgoing, trapped, and downgoing electron energy flux, respectively. (F-H)

153 Energy-time spectrograms of upgoing-to-perpendicular (up-to-perp, $f_{u} / f_{\perp}$ ), downgoing-to-

154 perpendicular (down-to-perp, $f_{d} / f_{\perp}$ ), and upgoing-to-downgoing $\left(f_{u} / f_{d}\right)$ electron energy flux, 155 respectively. Note in the $f_{d} / f_{\perp}$ spectrogram the clear decrease in the minimum energy of $f_{d} / f_{\perp} \sim 1$ 156 with increasing latitude, a characteristic signature of precipitation by field-line scattering ${ }^{11}$. (I-J)

157 Energy flux in channels HiE and LoE, respectively (black: $f_{\perp}$; blue: $f_{d}$; red: $f_{u}$ ). (K-L) Ratios of 158 energy flux in channels HiE and LoE, respectively (black: $f_{u} / f_{d} ;$ blue: $f_{d} / f_{\perp} ;$ red: $f_{u} / f_{\perp}$ ). Annotations 159 denote L-shell (L), dipole magnetic local time (MLT), dipole magnetic latitude (MLA), and 160 Universal Time (UT). 

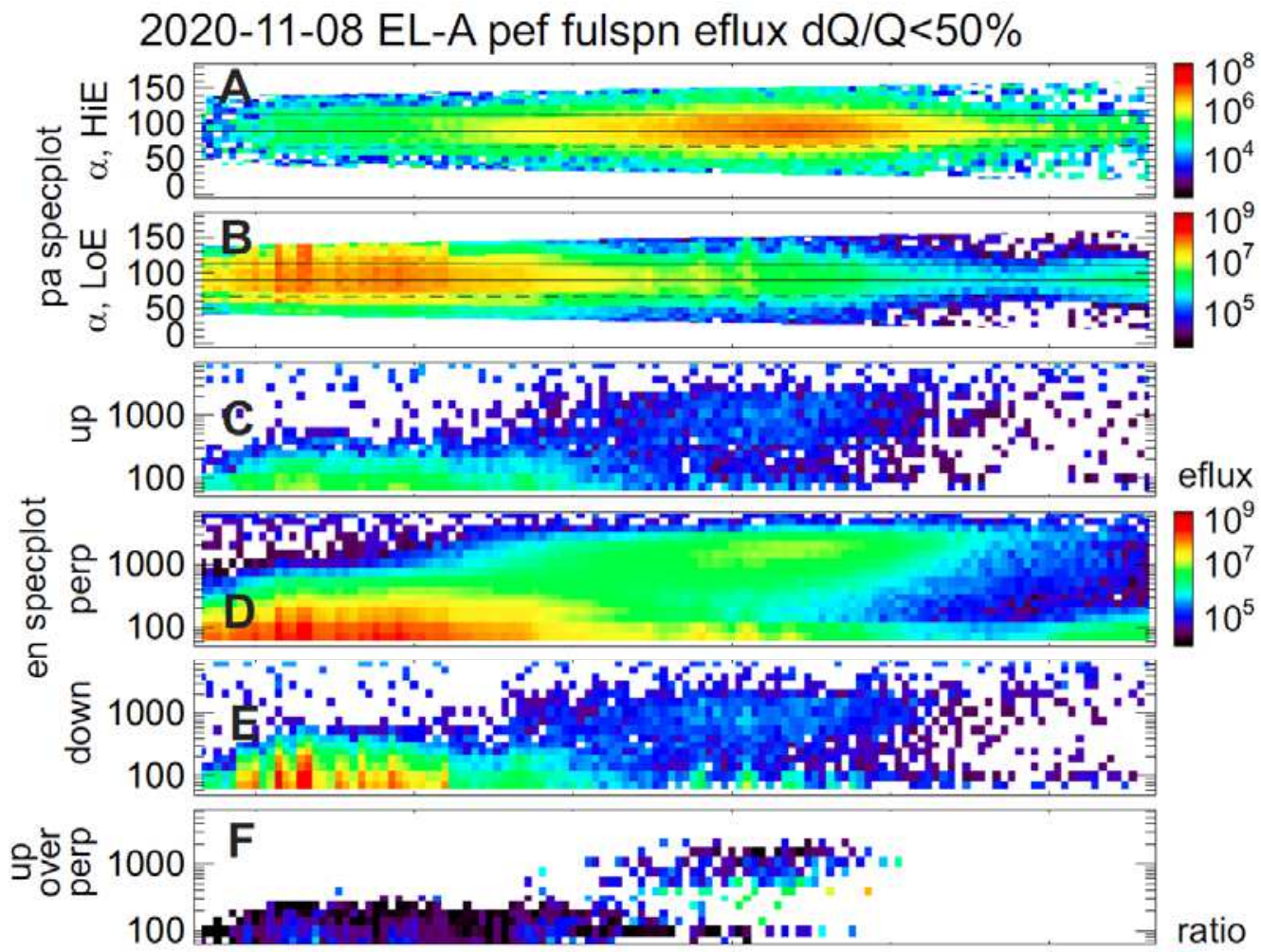

ratio
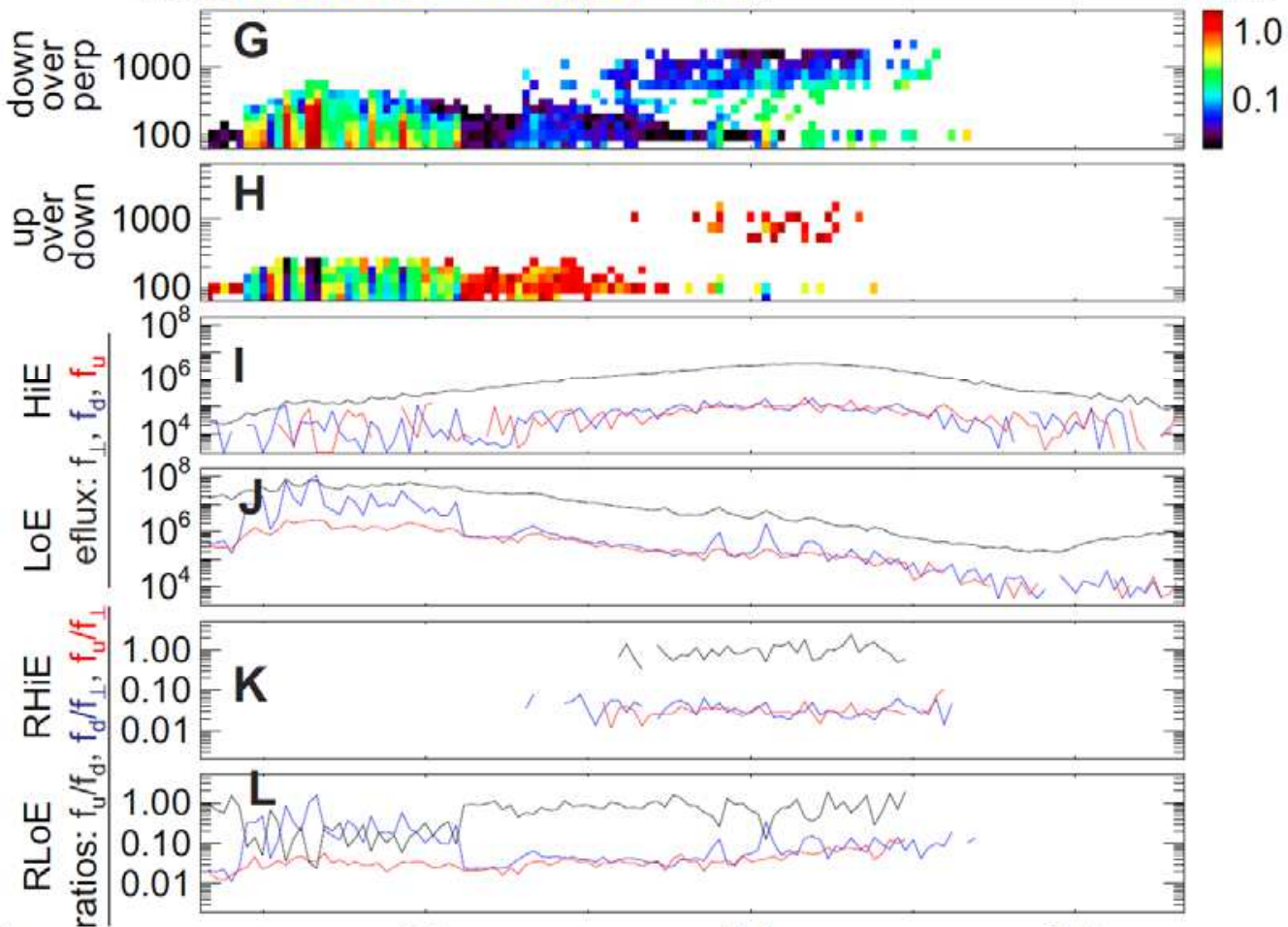

$\mathrm{M} L T$
MLA

166

hhmm
9.3

10.4

$-70.2$

0924
5.1

10.7

0926
3.3

11.0

$-55.2$ 
167 Extended Data Figure 2 | ELFIN-A dayside crossing of the outer radiation belt and auroral zone during the late recovery phase of a small storm. Format of Panels $A-L$ is identical to that

169 of Panels b-m in Figure 1. (A-B) Pitch-angle spectrograms of differential directional (broad)

170 electron energy flux channels $\mathrm{HiE}$ and LoE (430-5800keV and 50-430keV, respectively). Upper

171 solid and bottom dashed horizontal lines: loss cone $\left(\alpha=\alpha_{L C}\right)$ and anti-loss cone $\left(\alpha=180^{\circ}-\alpha_{L C}\right)$;

172 middle solid line: $\alpha=90^{\circ}$. (C-E) Energy-time spectrograms of upgoing, trapped, and downgoing

173 electron energy flux, respectively. (F-H) Energy-time spectrograms of upgoing-to-perpendicular

174 (up-to-perp, $f_{u} / f_{\perp}$ ), downgoing-to-perpendicular (down-to-perp, $f_{d} / f_{\perp}$ ), and upgoing-to-

175 downgoing $\left(f_{u} / f_{d}\right)$ electron energy flux, respectively. (I-J) Energy flux in channels HiE and LoE,

176 respectively (black: $f_{\perp}$; blue: $f_{d}$; red: $f_{u}$ ). (K-L) Ratios of energy flux in channels HiE and LoE,

177 respectively (black: $f_{u} / f_{d} ;$ blue: $f_{d} / f_{\perp} ;$ red: $f_{u} / f_{\perp}$ ). Annotations denote L-shell (L), dipole magnetic

178 local time (MLT), dipole magnetic latitude (MLA), and Universal Time (UT).

179

180 


\section{Extended Data Figure 3}

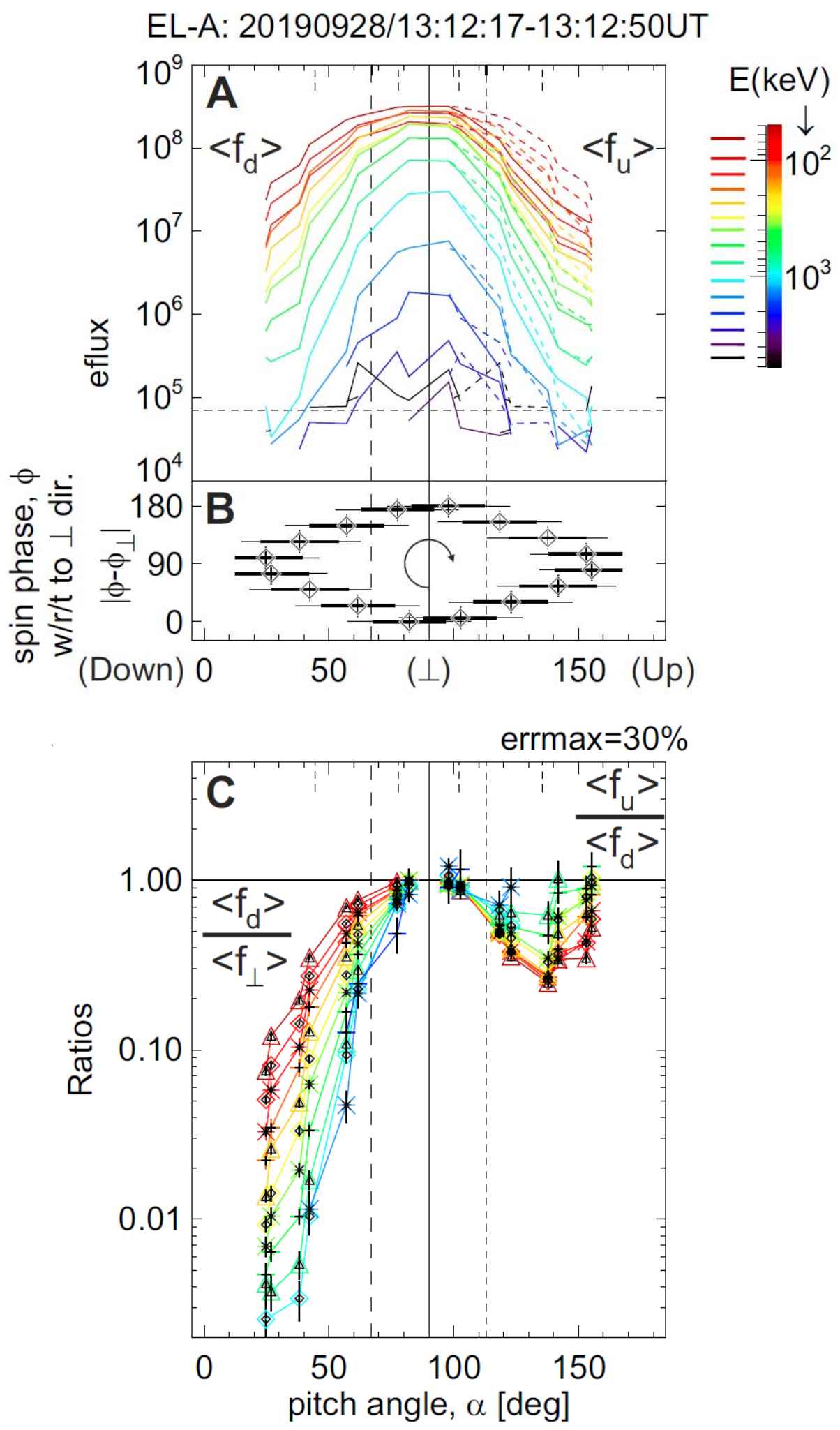

182 
183 Extended Data Figure 3 | Veracity of loss-cone fluxes and their ratios. (A) Pitch-angle spectra

184 of average fluxes from 11 spins (time interval indicated atop) for EPDE's logarithmically-

185 equidistant energy channels from low (warmer colors) to high (colder colors) for ELFIN-A.

186 Vertical lines denote pitch angles $\alpha=90^{\circ}$ (middle, solid), the loss cone ( $\alpha=\alpha_{L C}$, left, long-dashed),

187 and the anti-loss cone $\left(\alpha=180^{\circ}-\alpha_{L C}\right.$, right, short-dashed). Dotted colored lines denote

188 downgoing fluxes mirrored about pitch-angle $\alpha=0^{\circ}$ (i.e., plotted versus the supplementary of

189 their pitch angles) for easy comparison with upgoing fluxes at the same energy (solid colored

190 lines). The limits used to select field-aligned and perpendicular pitch-angle sector centers are

191 four short dashed lines hanging down from the top of the panel. Two are $22.5^{\circ}$ closer to the

192 field-line direction than the loss and the anti-loss cone, respectively; two are $11.25^{\circ}$ closer to

193 perpendicular than the loss and the anti-loss cone, respectively. (B) Sector pitch angle, $\alpha$

194 (center, diamond), and width (acceptance angle, horizontal bar) as function of the sector

195 center's spin-phase absolute distance from the (ascending) direction perpendicular to the

196 magnetic field, $\left|\phi-\phi_{\perp}\right|$. The arrow in the centered circle denotes the direction of the detector's

197 rotation in time during the spin. The thin horizontal bar centered at the diamond denotes the

198 sector's pitch-angle full-width full max; the thick bar denotes its full-width half max. (C) Pitch-

199 angle spectra of ratios of average fluxes for each energy channel (color) as determined from

200 Panel A. The down-to-perpendicular ratio is on the left $\left(0^{\circ}<\alpha<90^{\circ}\right)$; the upgoing-to-downgoing

201 ratio is on the right $\left(90^{\circ}<\alpha<180^{\circ}\right)$. Vertical dashed lines are same as in Panel A. Vertical bars at

202 each point demarcate $\pm d r$, the absolute error value for each ratio $r$. Only points with $d r / r<30 \%$

203 are shown.

204 

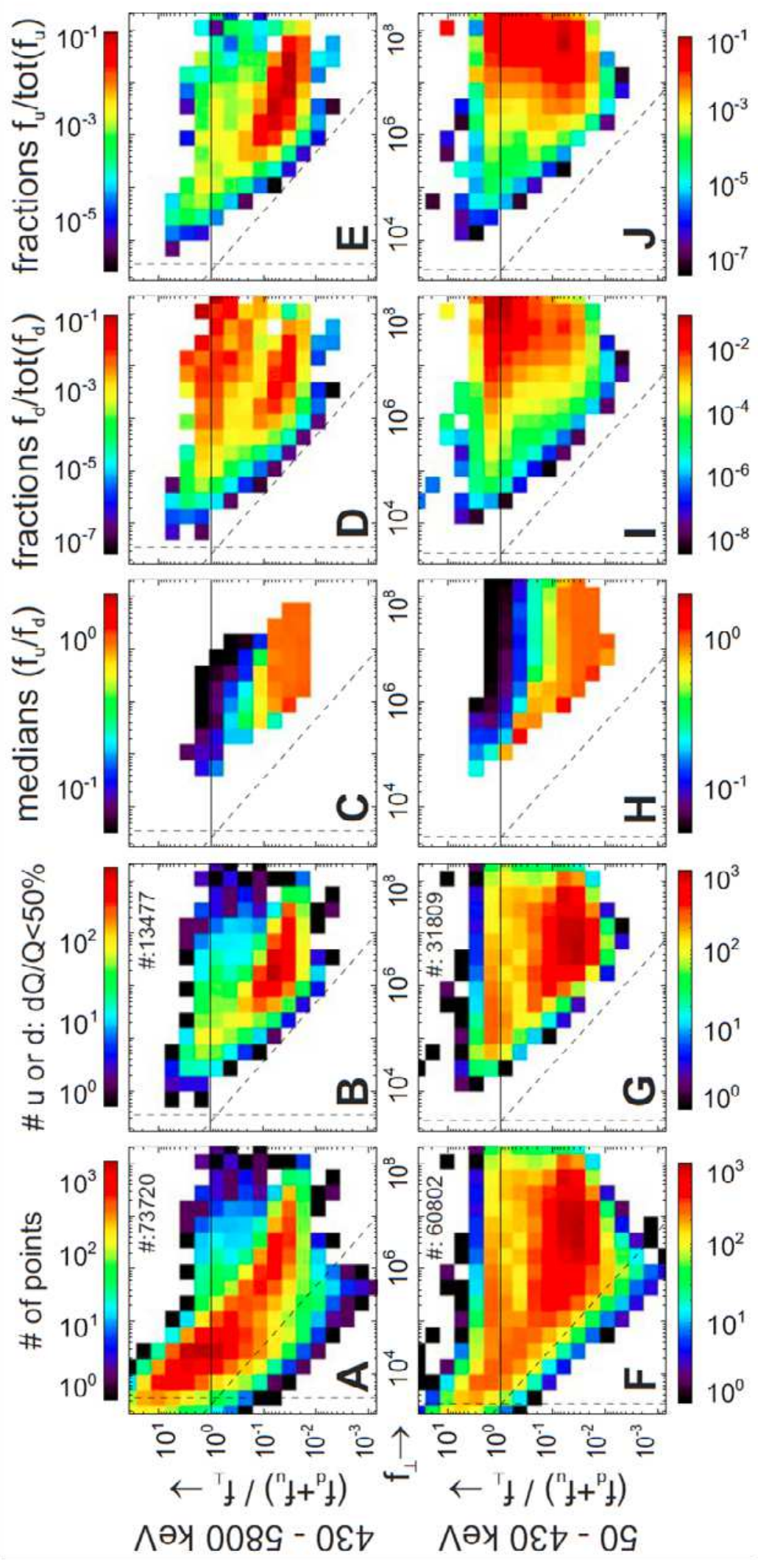

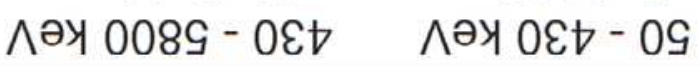


208 flux ratios. All panels show distributions in two-dimensional (2D) space $\left(f_{\perp},\left(f_{d}+f_{u}\right) / f_{\perp}\right)$, where $f_{\perp}$

209 is the differential directional energy flux (in $\mathrm{keV} / \mathrm{cm}^{2} \mathrm{~s}$ str MeV) measured near $\alpha=90^{\circ}$ (trapped

210 flux, perpendicular, $\perp$, to the $B$ field) and $\left(f_{d}+f_{u}\right)$ is the upward-plus-downward flux (in the loss

211 cone and anti-loss cone). Top and bottom rows are for the HiE and LoE channels (430-5800keV

212 and 50-430keV), respectively. Vertical dashed lines are the electronic noise flux values, $f_{n_{-} \text {HiE }}$

213 and $\mathrm{f}_{\mathrm{n} \_\mathrm{HiE}}$, for the HiE and LoE channels, respectively; diagonal dashed lines are the electronic

214 noise divided by $f_{\perp}$. Measurements to the left of these lines are consistent with electronic noise.

215 (A, F) Distribution of data in the database used (number of samples, \#, indicated as an insert).

216 (B, G) Distribution of data with statistically significant upgoing or downgoing fluxes $(\mathrm{df} / \mathrm{f}<50 \%)$,

217 which additionally eliminates samples corresponding to electronic noise. Note that most low $f_{\perp}$

218 points have been eliminated from flux ratios in these and remaining 2D panels in the figure;

219 averages computed from these statistically significant samples are intended to be

220 representations of the total measured flux for the purpose of computing flux ratios, not the

221 absolute flux. (Absolute flux depends on absolute detector efficiency, which has not yet been

222 fully evaluated, but is not critical for this study). Panels (B, G) are the $2 D$ versions of the PDFs

223 for HiE and LoE in Figure 3a. (C, H) Distribution of medians of ratios $f_{d} / f_{u}$ for statistically

224 significant fluxes. As $\left(f_{d}+f_{u}\right) / f_{\perp}$ decreases, most medians increase from a few $\%$ to $100 \%$ for

225 most $f_{\perp}$ values, particularly in cells with large numbers of points in Panels $B$ and G. Panels $(C, H)$

226 are the $2 \mathrm{D}$ versions of the median $\mathrm{f}_{u} / \mathrm{f}_{\mathrm{d}}$ lines in Figure 3a. $(\mathrm{D}, \mathrm{I})$ Distribution of the relative

227 contribution to the total downgoing flux, $f_{d}$, by each cell in this $2 D$ space. Two clusters of points

228 with very weak dependence on $f_{\perp}$ are evident: one near $\left(f_{d}+f_{u}\right) / f_{\perp} \sim 0.04$, which we attributed to 
229 atmospheric scattering of trapped particles, and another near $\left(f_{d}+f_{u}\right) / f_{\perp} \sim 1$, which we attributed

230 to magnetospheric scattering. These are the 2D versions of the line plots for HiE and LoE in

231 Figure 3c. (E, J) Same as in Panels $(D, I)$ except for the upgoing flux, $f_{u}$. The same two main

232 populations are evident here, as well. These are the $2 \mathrm{D}$ versions of the line plots for $\mathrm{HiE}$ and LoE

233 in Figure $3 b$.

234

235

236 


\begin{tabular}{|c|c|c|c|c|c|c|}
\hline \multicolumn{7}{|c|}{ Average Directional Electron Fluxes ${ }^{\dagger}$ and their Ratios } \\
\hline \multicolumn{7}{|c|}{ All data in $3<\mathrm{L}<7$} \\
\hline MLT & Energy [keV] & $r=\left\langle f_{u}\right\rangle /\left\langle f_{d}\right\rangle$ & $\left\langle f_{d}\right\rangle /\left\langle f_{\perp}\right\rangle$ & $\left\langle f_{\perp}\right\rangle$ [Units] & \multirow{2}{*}{\multicolumn{2}{|c|}{ Inferred ${ }^{\ddagger}$ Ratios }} \\
\hline \multirow{2}{*}{ Night } & LoE: $50-430$ & $9.3 \%$ & $35.1 \%$ & $1.65 \mathrm{E}+07 \mathrm{I}=\mathrm{\pi}$ & & \\
\hline & HiE: $430-5800$ & $27.8 \%$ & $8.3 \%$ & $1.50 \mathrm{E}+06$ & \multirow{4}{*}{ 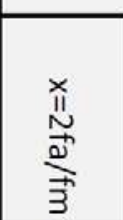 } & \multirow{4}{*}{ 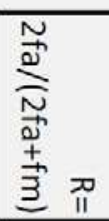 } \\
\hline \multirow{2}{*}{ Day } & LOE: $50-430$ & $21.4 \%$ & $16.3 \%$ & \multirow{4}{*}{ 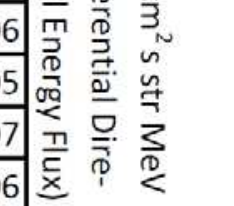 } & & \\
\hline & HiE: $430-5800$ & $81.2 \%$ & $4.9 \%$ & & & \\
\hline \multirow{3}{*}{$\begin{array}{l}\text { Night+Day, } \\
\text { Residence-ti- } \\
\text { me Norm'ed }\end{array}$} & LoE: $50-430$ & $11.1 \%$ & $30.0 \%$ & & & \\
\hline & $\mathrm{HiE}: 430-5800$ & $37.5 \%$ & $7.4 \%$ & & $120 \%$ & $55 \%$ \\
\hline & $50-5800$ (Integral) & $17.4 \%$ & $17.3 \%$ & $9.83 \mathrm{E}+06 \mathrm{keV} / \mathrm{cm}^{2} \mathrm{~s} \mathrm{str}$ & $42 \%$ & $30 \%$ \\
\hline \multicolumn{7}{|c|}{$\mathrm{D}_{\mathrm{ST}}<-20 \mathrm{nT}$ in $3<\mathrm{L}<7$} \\
\hline MLT & Energy [keV] & $r=\left\langle f_{u}\right\rangle /\left\langle f_{d}\right\rangle$ & $\left\langle f_{d}\right\rangle \mid\left\langle f_{\perp}\right\rangle$ & $\left\langle f_{\perp}\right\rangle$ [Units] & \multirow{2}{*}{\multicolumn{2}{|c|}{ Inferred ${ }^{\ddagger}$ Ratios }} \\
\hline \multirow{2}{*}{ Night } & LoE: $50-430$ & $8.5 \%$ & $35.7 \%$ & 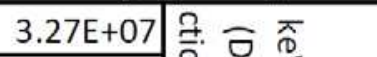 & & \\
\hline & HiE: $430-5800$ & $22.7 \%$ & $7.8 \%$ & $2.37 \mathrm{E}+06$ 票 $\leqslant$ & \multirow{4}{*}{ 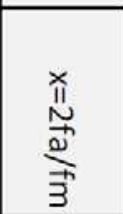 } & \multirow{4}{*}{ 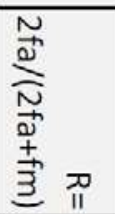 } \\
\hline \multirow{2}{*}{ Day } & LoE: $50-430$ & $23.2 \%$ & $12.5 \%$ & $1.16 \mathrm{E}+07 \mathrm{~m}$ & & \\
\hline & HiE: $430-5800$ & $90.4 \%$ & $3.9 \%$ & $5.96 \mathrm{E}+05 \stackrel{\mathbb{2}}{0} \cong$ & & \\
\hline \multirow{3}{*}{$\begin{array}{l}\text { Night+Day, } \\
\text { Residence-ti- } \\
\text { me Norm'ed }\end{array}$} & LoE: $50-430$ & $10.2 \%$ & $29.6 \%$ & $2.21 \mathrm{E}+07$ 끌 윽 3 & & \\
\hline & $\mathrm{HiE}: 430-5800$ & $30.3 \%$ & $7.0 \%$ & $1.48 \mathrm{E}+06$ 玄 $\stackrel{0}{<}$ & $87 \%$ & $46 \%$ \\
\hline & $50-5800$ (Integral) & $13.8 \%$ & $18.6 \%$ & $1.64 \mathrm{E}+07 \mathrm{keV} / \mathrm{cm}^{2} \mathrm{~s} \mathrm{str}$ & $32 \%$ & $24 \%$ \\
\hline
\end{tabular}

Extended Data Table 1 | Differential and integral directional energy fluxes and ratios for the

240 outer radiation belt $(3<L<7)$ under all geomagnetic conditions (top) and for active $\left(D_{S T}<-20 n T\right)$

241 times (bottom). Ratios of time averages rather than medians of ratios have been used to

242 accurately characterize total energy flux ratios. The combination of Night and Day ratios was

243 determined directly from the numbers above them, assuming equal satellite residence time in

244 the dayside and the nightside. The bottom row, which represents the integral directional

245 energy flux channel (50-5800keV), was computed directly from the rows above it. $f_{u}$ and $f_{d}$ are

246 upgoing and downgoing fluxes, <> represent averages, and $f_{a}$ and $f_{m}$ are the measured

247 contributions to precipitation from atmospheric scattering and magnetospheric scattering. The 
248 ratios $\mathrm{x}$ and $\mathrm{R}$ are the inferred net contributions to precipitation from atmospheric scattering

249 relative to magnetospheric scattering and relative to the total precipitation, respectively. 

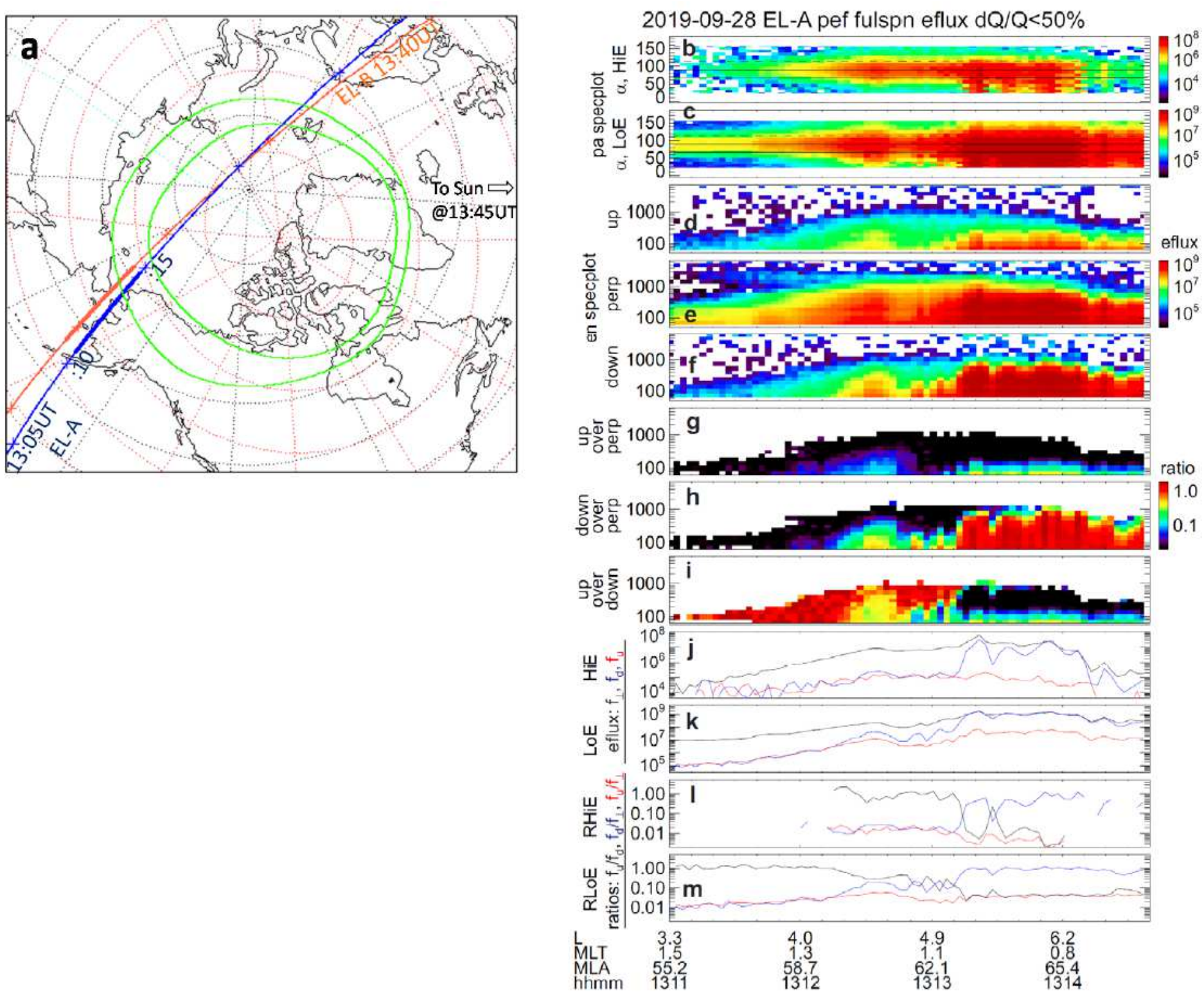

\section{Figure 1}

ELFIN storm $\Downarrow$ time, nightside crossing of the outer radiation belt and auroral zone. Please see manuscript .pdf for full caption. Note: The designations employed and the presentation of the material on this map do not imply the expression of any opinion whatsoever on the part of Research Square concerning the legal status of any country, territory, city or area or of its authorities, or concerning the delimitation of its frontiers or boundaries. This map has been provided by the authors. 
Directional differential energy flux ratios

Red: $u / \perp$; blue: d/ $\perp$; black: $u / d$. Dashed: - - - Low AE; Solid: - High AE

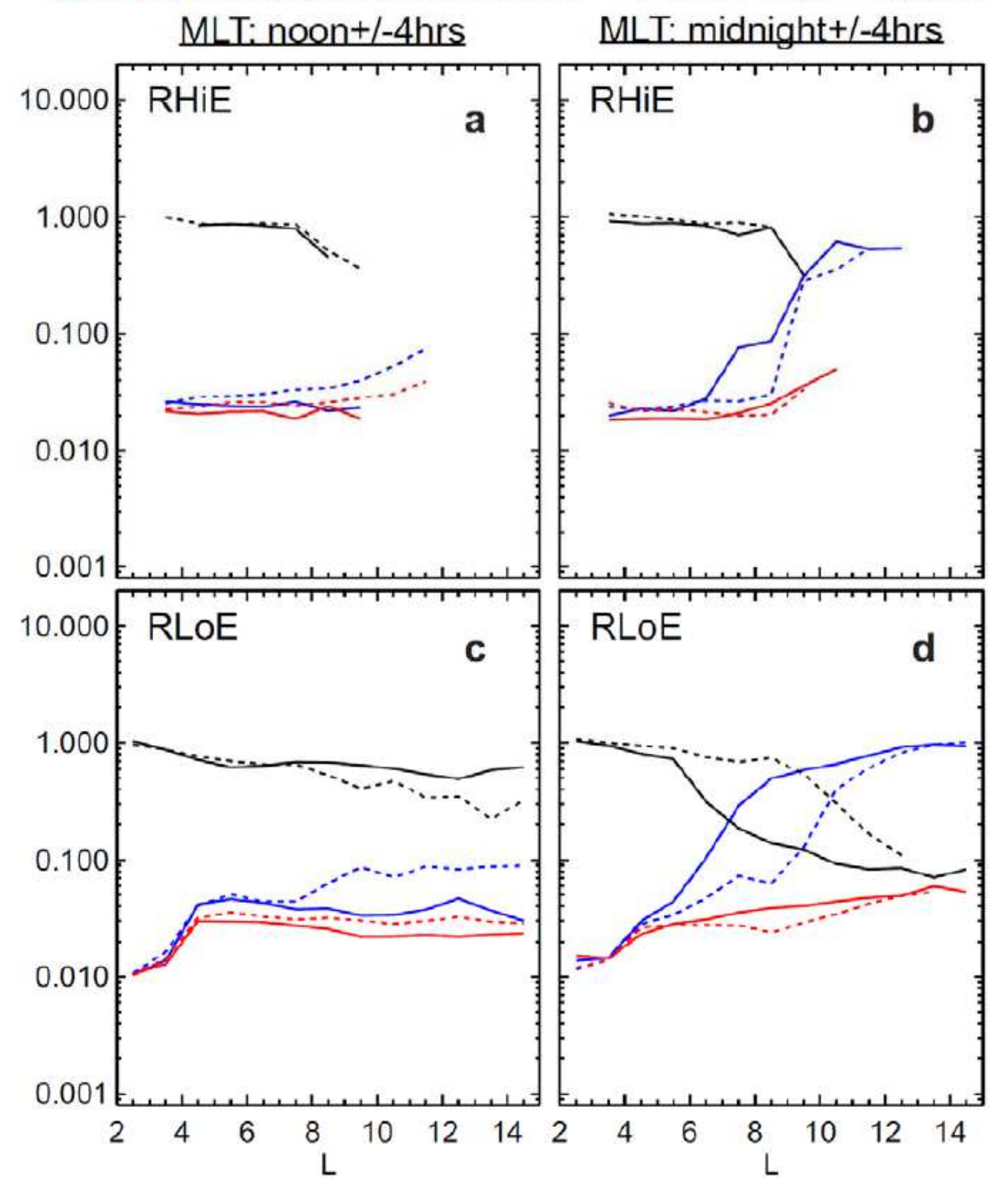

Figure 2

Dependence of energy flux ratios on L囚shell, local time, and activity. Please see manuscript .pdf for full caption. 

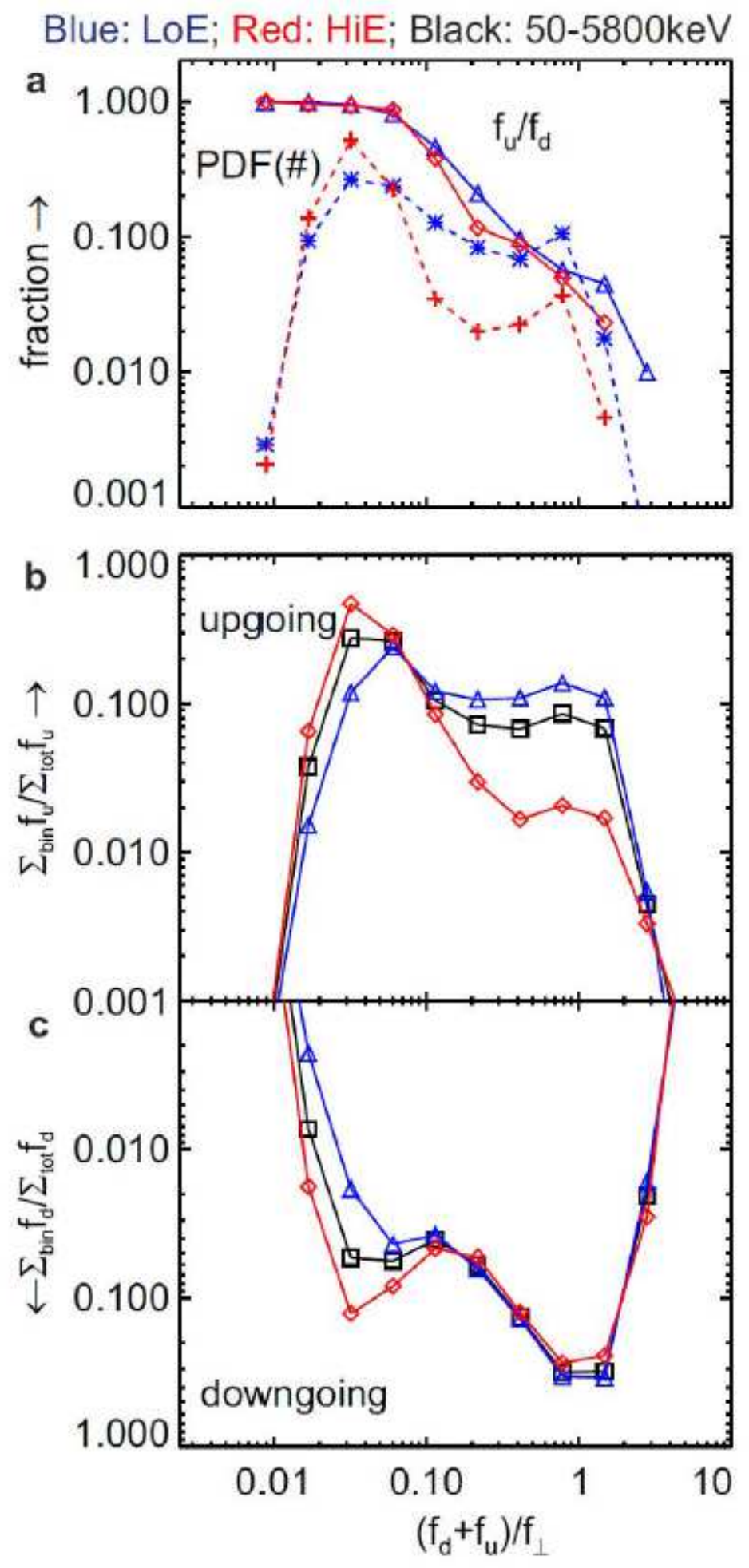

Figure 3

Distribution of data, fluxes, and flux ratios as a function of loss $₫$ cone flux. (a) Probability density functions of all data in the 150 and 1430 channels (blue stars and red crosses, respectively) and medians of flux ratios ( $\mathrm{fu} / \mathrm{fd}$ ) for these channels (blue triangles and red diamonds, respectively). (b) Relative contribution to net upward flux within the 150 and 1430 broad differential broad energy channels (blue triangles and red diamonds, respectively) and within the summed energy channel representing the total 
energy flux measured by the detector, i.e., at energies $50 \mathrm{keV}-5.8 \mathrm{MeV}$ (black squares). (c) Same as in (c) but for the downward flux. Two®dimensional versions of several of these distributions, also plotted against the perpendicular energy flux, are shown in Extended Data Figure 4. 